\title{
Relaxation Dynamics of an Isolated Large-Spin Fermi Gas Far from Equilibrium
}

\author{
Ulrich Ebling, ${ }^{1, *}$ Jasper Simon Krauser, ${ }^{2}$ Nick Fläschner, ${ }^{2}$ Klaus Sengstock, ${ }^{2,3}$ Christoph Becker, ${ }^{2,3}$ \\ Maciej Lewenstein, ${ }^{1,4}$ and André Eckardt ${ }^{5}$ \\ ${ }^{1}$ ICFO_Institut de Ciències Fotòniques, Avenida Carl Friedrich Gauss, \\ 3, E-08860 Castelldefels (Barcelona), Spain \\ ${ }^{2}$ Institut für Laserphysik, Universität Hamburg, Luruper Chaussee 149, D-22761 Hamburg, Germany \\ ${ }^{3} \mathrm{ZOQ}$-Zentrum für optische Quantentechnologien, Universität Hamburg, \\ Luruper Chaussee 149, 22761 Hamburg, Germany \\ ${ }^{4}$ ICREA-Institució Catalana de Recerca i Estudis Avançats, \\ Lluís Companys 23, E-08010 Barcelona, Spain \\ ${ }^{5}$ Max-Planck-Institut für Physik komplexer Systeme, Nöthnitzer Straße 38, D-01187 Dresden, Germany
} (Received 3 January 2014; published 16 April 2014)

\begin{abstract}
A fundamental question in many-body physics is how closed quantum systems reach equilibrium. We address this question experimentally and theoretically in an ultracold large-spin Fermi gas where we find a complex interplay between internal and motional degrees of freedom. The fermions are initially prepared far from equilibrium with only a few spin states occupied. The subsequent dynamics leading to redistribution among all spin states is observed experimentally and simulated theoretically using a kinetic Boltzmann equation with full spin coherence. The latter is derived microscopically and provides good agreement with experimental data without any free parameters. We identify several collisional processes that occur on different time scales. By varying density and magnetic field, we control the relaxation dynamics and are able to continuously tune the character of a subset of spin states from an open to a closed system.
\end{abstract}

DOI: 10.1103/PhysRevX.4.021011

\section{INTRODUCTION}

The relaxation of closed quantum systems toward equilibrium is a fundamental problem in many-body physics. It is particularly challenging to fully understand this macroscopic process on the basis of microscopic properties [1-4]. Here, ultracold atomic quantum gases provide an exceptional experimental platform due to the nearly perfect isolation from their environment and the excellent control on a microscopic level. In particular, the possibility to prepare well-defined states far from equilibrium, as well as widely tunable Hamiltonians, has recently attracted a lot of attention; e.g., prethermalization [5-7], relaxation in strongly interacting lattice systems [8-12], and the interplay between thermal and condensate fractions of multicomponent bosons have been studied [13].

Spinor quantum gases are of particular interest since the spin offers an additional degree of freedom, giving rise to complex dynamics involving different relaxation processes

*ulrich.ebling@icfo.es

Published by the American Physical Society under the terms of the Creative Commons Attribution 3.0 License. Further distribution of this work must maintain attribution to the author(s) and the published article's title, journal citation, and DOI.
Subject Areas: Atomic and Molecular Physics, Condensed Matter Physics

on different time scales. Ultracold bosonic quantum gases have been intensively studied and exhibit a rich variety of effects such as texture formation and spin dynamics in spinor Bose-Einstein condensates (BECs) [14-18], which can be well described theoretically using a multicomponent Gross-Pitaevskii equation [19-21]. Recently, collective spin dynamics was also observed in a thermal Bose gas [22]. Fermions, in contrast, are governed by Pauli blocking and reveal a different behavior. So far, most experiments studied spin- $1 / 2$ fermions, e.g., the BEC-BCS crossover $[23,24]$, thermodynamic and transport properties [25,26], collective excitations [27,28], and magnetic ordering [29-32]. Spin-related phenomena in multicomponent Fermi gases $(F>1 / 2)$ have only recently been investigated, in the context of spin-mixing dynamics on individual sites of an optical lattice [33] or collective coherent excitations in a trapped system [34-36]. The latter has been proven to be well described within a Boltzmann equation. The question of how a large-spin fermionic many-body system reaches an equilibrium state via relaxation involving spin and spatial degrees of freedom has not been addressed.

In this paper, we study the relaxation dynamics of a trapped fermionic quantum gas of ${ }^{40} \mathrm{~K}$ atoms with a large spin of $F=9 / 2$. Starting from an initial mixture with only a few spin states occupied, we observe a rich relaxation 
dynamics that leads to a redistribution of the atoms among all available spin states. We study the intermediate regime between the collisionless and the hydrodynamic limits. In the collisionless limit, interactions are weak and can be taken into account on a mean-field level, while the hydrodynamic limit is characterized by stronger interactions that ensure local equilibrium. The dynamics in this intermediate regime is governed by different processes on very different time scales. We identify these processes by deriving a Boltzmann equation from the microscopic Hamiltonian of the large-spin system. This approach describes the time evolution of the system on the level of single particles in contact with the bath of the many-body system [37,38], which corresponds to the intuitive expectation that the system acts as a bath for its own subsystems.

We present a detailed comparison between numerical simulations and experimental data and find good agreement. Our analysis includes the dependence of the relaxation on density as well as on magnetic field. Whereas a higher density enhances the spin relaxation, we find a suppression of spin-changing processes at large magnetic fields due to the quadratic Zeeman shift. The latter effect can be used to control the loss of particles from the subsystem defined by the initially occupied spin components into the initially empty spin states. Generally, we observe that the relaxation within a subset of spin states, driven by incoherent spin-conserving collisions, happens on a much faster time scale than the redistribution among the spin components due to spin-changing incoherent collisions. The reason is that the spin-changing collisions are driven by the relatively small part of the interactions that breaks the $S U(10)$ symmetry between the spin states. Thus, we encounter a situation similar to prethermalization [7], where first a prethermal state is reached, approximately conserving the initial occupations of the single spin states, before the redistribution among all spin states due to slight symmetry breaking sets in. This separation of time scales also allows us to monitor the increase of (effective) temperature within the subsystem of the initially populated spin states, as it is caused by dissipation into empty spin states.

\section{RELAXATION PROCESSES IN A LARGE-SPIN SYSTEM}

We perform measurements in a quantum degenerate gas of fermionic ${ }^{40} \mathrm{~K}$, which has total spin of $F=9 / 2$ in its hyperfine ground state, yielding ten spin states $m=-9 / 2, \ldots,+9 / 2$, as depicted in Fig. 1(a). We prepare an atomic sample with two spin states occupied (see Appendix A 1 for details), confined in a spin-independent dipole trap. Because of the broken $S U(N)$ symmetry in ${ }^{40} \mathrm{~K}$ resulting from spin-dependent scattering lengths, spinchanging collisions can occur. A microscopic collision process is depicted in Fig. 1(b): Two particles collide and exchange both spin $m$ and momentum $k$ : $\left(\left|m_{1}, k_{1}\right\rangle+\left|m_{2}, k_{2}\right\rangle \rightarrow\left|m_{1}^{\prime}, k_{1}^{\prime}\right\rangle+\left|m_{2}^{\prime}, k_{2}^{\prime}\right\rangle\right)$. The total spin $S$, the total magnetization $M=m_{1}+m_{2}$, as well as the total momentum $k_{1}+k_{2}$ have to be conserved in this process. As a particular fermionic feature, the Pauli exclusion principle has to be obeyed, i.e., $m_{1} \neq m_{2}$ and $m_{1}^{\prime} \neq m_{2}^{\prime}$. The interplay between the differential quadratic Zeeman energy $\propto m_{1}^{2}+m_{2}^{2}-m_{1}^{\prime 2}-m_{2}^{\prime 2}$ and the interaction energy determines whether spin-changing collisions are likely or suppressed. In the presence of spin-changing collisions, the atoms will, in general, relax into a steady state with a population in all ten spin states. Hence, preparation of an initial nonequilibrium state with only a few spin states populated will lead to complex dynamics, in which more and more spin states are gradually occupied [see Fig. 1(c)]. How the system relaxes toward a steady state is a compelling question. (a)

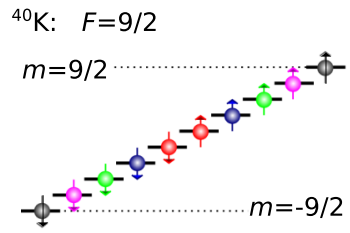

(b)

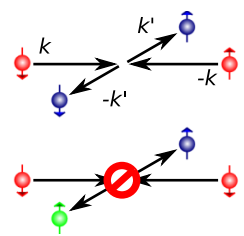

(c)
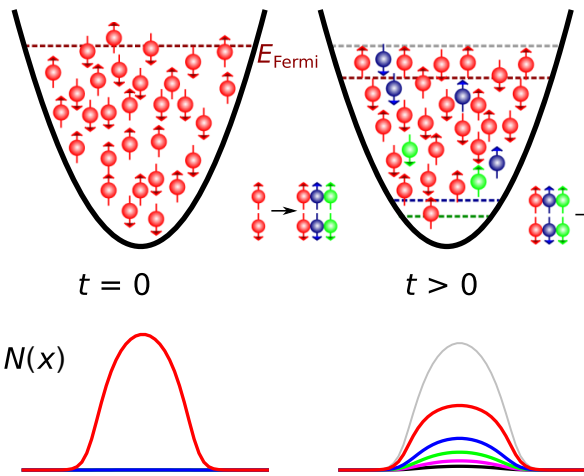

$t>0$

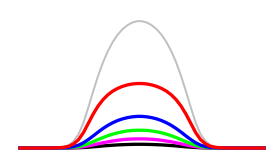

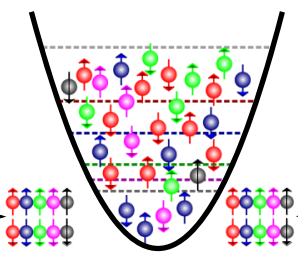

$t \gg 0$

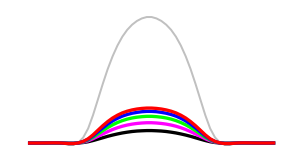

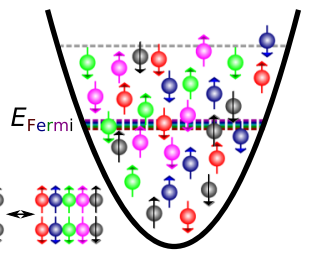

$t \rightarrow \infty$

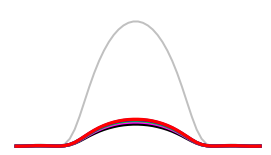

FIG. 1. Schematic description of the relaxation process in a large-spin Fermi gas involving spin and spatial degrees of freedom. (a) The ten spin states of ${ }^{40} \mathrm{~K}$. (b) A typical spin-changing collision in the center-of-mass frame and another collision forbidden by the Pauli exclusion principle. (c) Top: Initially, all atoms are prepared in a binary spin mixture $m= \pm 1 / 2$. Spin-changing collisions distribute atoms among all other spin states until an approximately balanced population is reached. The Fermi energies for each two-component subsystem are lower than the initial Fermi energy. Bottom: Time evolution of the spatial density for each spin component. 


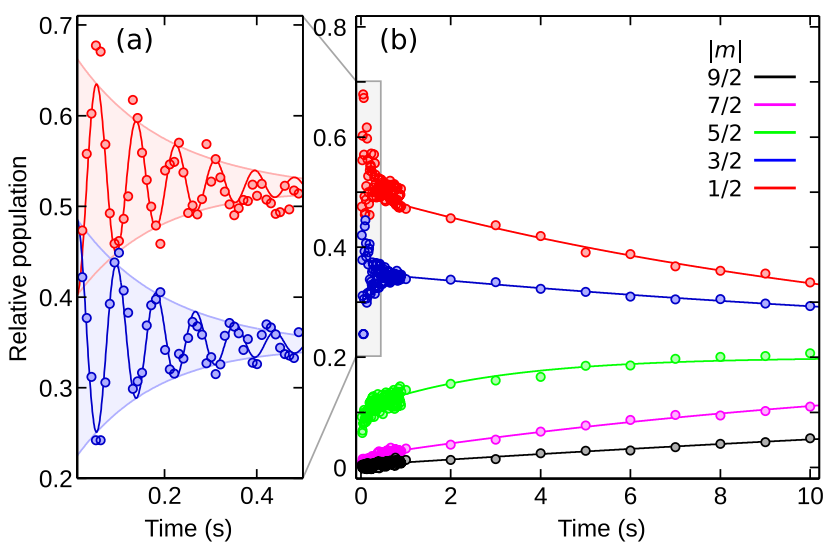

FIG. 2. (a) Measurement of damped spin oscillations and (b) subsequent relaxation toward equilibrium, observed in a $3 \mathrm{D}$ fermionic quantum gas with large spin. Depicted is the time evolution of the relative populations of all spin components $\pm m$, starting from an initial superposition of all ten spin states. For the exact experimental configuration, see Appendix A 1. Solid lines are guides to the eye. Note the three time scales of (i) the spin oscillations, (ii) their damping, and (iii) the subsequent relaxation of the total system. The redistribution among all spin states occurs on a time scale of $10 \mathrm{~s}$. The magnetic field is $B=0.17 \mathrm{G}$, particle number $N=4.9 \times 10^{5}$, and temperature $T / T_{F}=0.22$.

In Fig. 2, we show exemplarily an experimentally obtained time evolution of the spin occupations in our system. Here, the initial spin configuration is a superposition of all ten spin states created by rotating a mixture of the states $m= \pm 1 / 2$ using rf pulses [36]. We can clearly identify three different processes occurring on three different time scales: (i) We observe coherent spin-changing oscillations with a periodicity on the order of a hundred milliseconds. (ii) These oscillations are damped with a rate on the order of several hundreds of milliseconds. (iii) We also observe a slow redistribution among the ten spin states on a much longer time scale on the order of tens of seconds.

In the following, we derive a Boltzmann equation that reproduces the experimentally observed effects and enables us to distinguish which scattering processes are responsible for each effect. We show that the coherent oscillations (i) are a mean-field effect driven by forward scattering, where $\left\{k_{1}, k_{2}\right\}=\left\{k_{1}^{\prime}, k_{2}^{\prime}\right\}$. Their damping (ii) is dominated by spin-conserving nonforward collisions $\left\{k_{1}, k_{2}\right\} \neq$ $\left\{k_{1}^{\prime}, k_{2}^{\prime}\right\}$ and $\left\{m_{1}, m_{2}\right\}=\left\{m_{1}^{\prime}, m_{2}^{\prime}\right\}$ that lead to a momentum redistribution within the Fermi sea without changing the spin configuration. The long-term redistribution (iii) is governed by nonforward spin-changing collisions that change the momentum distribution within the Fermi sea as well as the spin configuration $\left\{k_{1}, k_{2}\right\} \neq\left\{k_{1}^{\prime}, k_{2}^{\prime}\right\}$ and $\left\{m_{1}, m_{2}\right\} \neq\left\{m_{1}^{\prime}, m_{2}^{\prime}\right\}$.

The above-mentioned Boltzmann equation includes all these collision processes and captures the nonequilibrium dynamics in a general fashion, applicable to trapped weakly interacting gases with arbitrary spin. In this approach, the single-particle dynamics is treated as an open system in contact with the environment represented by all the other particles. An approach for deriving a Boltzmann equation was applied successfully to the description of spin dynamics in liquid hydrogen and helium [39-45] and later in spin-1/2 Fermi gases [46,47]. In this paper, we generalize this approach to one- and three-dimensional systems with large spin, accounting for the quadratic Zeeman effect (QZE). In general, a kinetic equation or Boltzmann equation is used to describe the time evolution of the single-particle density matrix $\hat{\rho}$. It has the form

$$
\frac{d}{d t} \hat{\rho}-\frac{1}{i \hbar}\left[\hat{\rho}, \hat{H}_{0}\right]=I_{\text {coll }}[\hat{\rho}] .
$$

Here, $\hat{H}_{0}$ denotes the single-particle Hamiltonian

$$
\hat{H}_{0}=\frac{\hat{p}^{2}}{2 M}+\frac{1}{2} M \omega^{2} \hat{x}^{2}+Q \hat{S}_{z}^{2},
$$

which contains the kinetic energy, the harmonic trapping potential, and the quadratic Zeeman splitting $Q$ induced by a homogeneous magnetic field. The term $I_{\text {coll }}[\hat{\rho}]$ on the right-hand side of Eq. (1) is called the collision term and is derived from two-particle contact interaction. Because of total spin conservation, collisions are best described in the basis of total spin $|S, M\rangle=$ $\sum_{m_{1}, m_{2}}\left|m_{1}, m_{2}\right\rangle\left\langle m_{1} m_{2} \mid S M\right\rangle$ with the short notation for Clebsch-Gordan coefficients $\quad\left\langle m_{1} m_{2} \mid S M\right\rangle \equiv$ $\left\langle F, m_{1} ; F, m_{2} \mid S, M\right\rangle$ that we use throughout this paper. In general, scattering in each channel of total spin $S$ depends on a different $s$-wave scattering length $a_{S}$. Because of antisymmetrization of the total wave function, $s$-wave scattering with odd $S$ is forbidden. Thus, for ${ }^{40} \mathrm{~K}$, there are five different scattering lengths present for $S=0,2,4,6,8$ [33]. In each collision channel defined by $|S, M\rangle$, particles interact with a contact interaction of strength $g_{S}^{3 \mathrm{D}}=\left(4 \pi \hbar^{2} / M\right) a_{S}$, which is used in all $3 \mathrm{D}$ calculations. We also consider a 1D system, in which the motion in two transversal directions is frozen out completely by a tight trapping potential (characterized by radial frequencies $\omega_{x, y}$ ) such that the effective $1 \mathrm{D}$ contact-interaction parameter is given by $g_{S}^{1 \mathrm{D}}=$ $2 \hbar \sqrt{\omega_{y} \omega_{z}} a_{S}$ (see Appendix A 1 for details). The notation $g_{S} \equiv g_{S}^{1 \mathrm{D}}$ for this quantity is used throughout this paper. In ${ }^{40} \mathrm{~K}$, the $a_{S}$ range from 120 to $170 \mathrm{Bohr}$ radii.

We obtain an explicit expression for the collision term in Eq. (1) using the method originally developed by Lhuillier and Laloë $[43,44,46]$ for transport properties in helium. In this approach, collisions are treated as single "atomic beam" experiments, where the colliding particles are assumed to be uncorrelated before and after a collision, reminiscent of Boltzmann's original molecular chaos hypothesis, but the scattering process itself is treated on a full quantum level. This approximation is valid for dilute 
gases where the mean time between collisions is long and the particle number is large. In this regime, binary collisions can be described by the $T$-matrix, which connects the two-body density matrices before and after a collision. Subsequently, the description is reduced to a singleparticle level by tracing out the second particle, similar to tracing out a thermal bath in studies of collisional decoherence $[37,38]$.

We calculate the kinetic equation (1) in its phase-space representation, where the single-particle density matrix $\rho_{m n}\left(x, x^{\prime}\right)$ is expressed by the Wigner function

$$
W_{m n}(x, p)=\int \frac{d y}{2 \pi \hbar} e^{i p y / \hbar} \rho_{m n}\left(x+\frac{y}{2}, x-\frac{y}{2}\right) .
$$

Note that we perform the transformation only with respect to the spatial degrees of freedom. With respect to spin, denoted by the indices, it retains the form of a singleparticle density matrix. The derivation is carried out in detail in Sec. IV and involves a semiclassical gradient expansion of the Wigner function in position and momentum space, leading to an equation in matrix form given by

$$
\begin{aligned}
\frac{d}{d t} W(x, p)+\partial_{0} W(x, p)+\frac{i}{\hbar}\left[Q S_{z}^{2}+V^{\mathrm{MF}}(x), W(x, p)\right] \\
-\frac{1}{2}\left\{\partial_{x} V^{\mathrm{MF}}(x), \partial_{p} W(x, p)\right\}=I_{\text {coll }}(x, p),
\end{aligned}
$$

where the collision integral reads

$$
\begin{aligned}
I_{m n}^{\text {coll }}(x, p)= & -\frac{M}{\hbar^{2}} \sum_{a b l}\left\{\int_{q^{2}>\epsilon_{1}} d q \frac{\tilde{U}_{\text {malb }}}{\sqrt{q^{2}+\Delta_{\text {mlab }}}} W_{a n}(x, p) W_{b l}(x, p-q)+\int_{q^{2}>\epsilon_{2}} d q \frac{\tilde{U}_{\text {nalb }}}{\sqrt{q^{2}+\Delta_{\text {nlab }}}} W_{m a}(x, p) W_{l b}(x, p-q)\right\} \\
& +\frac{M}{\hbar^{2}} \sum_{a b c d l} \int_{q^{2}>\epsilon_{3}} d q \frac{U_{\text {malb }} U_{\text {ncld }}}{\sqrt{q^{2}+\Delta_{\text {mnlabcd }}}} W_{a c}\left(x, p-\frac{1}{2}\left(q-\sqrt{q^{2}+\Delta_{\text {mnlabcd }}}\right)\right) W_{b d}\left(x, p-\frac{1}{2}\left(q+\sqrt{q^{2}+\Delta_{\text {mnlabcd }}}\right)\right) .
\end{aligned}
$$

Here, we define the coupling constants

$$
\begin{gathered}
U_{a c b d}=\sum_{S, M} g_{S}\langle a b \mid S M\rangle\langle S M \mid c d\rangle, \\
\tilde{U}_{a c b d}=\sum_{S, M} g_{S}^{2}\langle a b \mid S M\rangle\langle S M \mid c d\rangle
\end{gathered}
$$

and denote energy shifts induced by the quadratic Zeeman splitting as $\Delta_{a b c d}=4 M Q\left(a^{2}+b^{2}-c^{2}-d^{2}\right)$ and $\Delta_{\text {mnlabcd }}=$ $2 M Q\left(m^{2}+n^{2}+2 l^{2}-a^{2}-b^{2}-c^{2}-d^{2}\right)$. The infrared cutoffs are given by $\epsilon_{1}=\left(M U_{\text {malb }} / \hbar\right)-\Delta_{\text {mlab }}, \epsilon_{2}=$ $\left(M U_{\text {nalb }} / \hbar\right)-\Delta_{\text {nlab }}, \quad$ and $\quad \epsilon_{3}=M U_{\text {nalb }} / 2 \hbar\left(U_{\text {malb }}+\right.$ $\left.U_{\text {ncld }}\right)-\Delta_{\text {mnlabcd }}$.

Equation (4) contains several terms, each describing a different dynamical process. The free particle motion in the trap is described by $\partial_{0}=p / M \partial_{x}-M \omega_{x}^{2} x \partial_{p}$. The leading interaction term appears in the commutator $[\cdot, \cdot]$. The commutator drives coherent spin dynamics through the interplay of the quadratic Zeeman effect and a spindependent mean-field potential resulting from forward scattering:

$$
V_{m n}^{\mathrm{MF}}(x)=2 \sum_{a b} U_{m n a b} N_{a b}(x),
$$

a function of the density $N(x)=\int d p W(x, p)$. In our largespin system, described by several scattering lengths $a_{S}$, it is helpful to decompose the mean-field potential [Eq. (8)] into two contributions. The first contribution is symmetric with respect to all $N$ spin states and proportional to the mean scattering length. It conserves the occupations of the different spin components. The second term contains that part of the interactions that breaks the $S U(N)$ symmetry between the spin states and describes spin-changing processes. It depends on differences of scattering lengths only and is, thus, typically much smaller than the symmetric term. The commutator in Eq. (4) vanishes unless the Wigner function possesses off-diagonal elements indicating spin coherence. Moreover, the symmetric spin-conserving mean-field interactions can only contribute if the Wigner function describes an inhomogeneous spin state.

The mean-field potential also appears in the anticommutator $\{\cdot, \cdot\}$. This term results from the subleading order of the semiclassical gradient expansion (where the spinindependent trapping potential also appears) and it is generally smaller than the commutator. It describes spindependent forces that modify the kinetics in the trap.

The collision integral [Eq. (5)] describes effects beyond the mean field that result from nonforward scattering and generates a dynamics that appears incoherent on the level of a single-particle description. It is quadratic in the scattering lengths. Again, we have to distinguish between $S U(N)$ symmetric spin-conserving collision processes on the one hand and spin-changing collisions on the other. The latter processes are described by those terms for which the quadratic Zeeman shifts $\Delta$ are nonzero and they are much smaller than the former, since they depend only on the relatively small differences between the scattering lengths.

The 1D equation (4) allows for a numerical treatment with standard methods. An exemplary result is depicted in Fig. 3 and shows the relaxation dynamics starting from the 


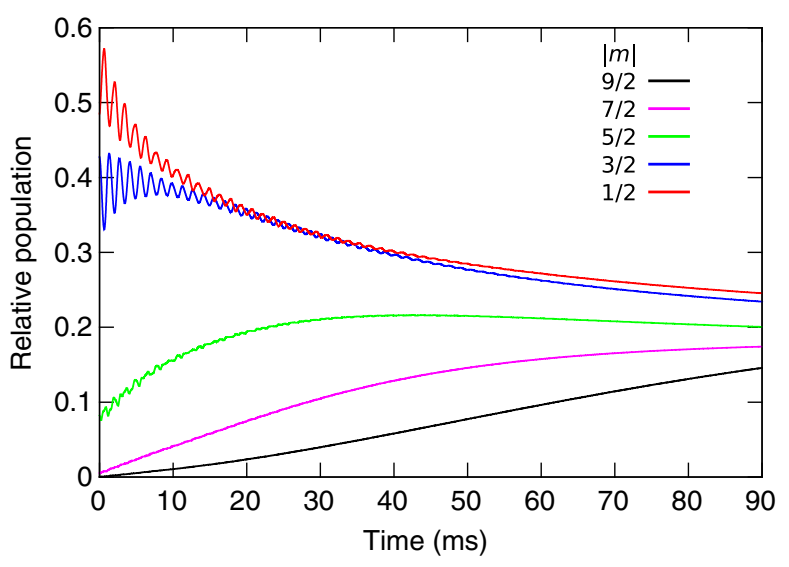

FIG. 3. Numerical simulation of coherent oscillations, damping, and relaxation in the $1 \mathrm{D}$ case. The initial spin configuration is the same as in Fig. 2. The axial trapping frequency is $\omega_{x}=2 \pi \times 84 \mathrm{~Hz}$, and radial frequencies are $\omega_{y, z}=2 \pi \times 47 \mathrm{kHz}$, particle number $N=100$ per tube at temperature $T / T_{F}=0.2$, and magnetic field $B=1.5 \mathrm{G}$. As in Fig. 2, we observe three time scales related to oscillations, damping, and relaxation.

same initial superposition as in Fig. 2, but in a 1D setup. The comparison of the two figures allows us to assign each of the three different processes visible to one of the terms in the Boltzmann equation: (i) The coherent oscillations are driven by the commutator in Eq. (4), which is linear in differences of scattering lengths and describes forward collisions. (ii) The damping of coherent phenomena arises from the spinconserving part of the collision integral [Eq. (5)], which is quadratic in the scattering lengths. We have checked that spatial dephasing is not responsible, as it is suppressed by the dynamically induced long-range nature of mean-field interactions induced by the rapid particle motion in the trap $[36,48]$. (iii) The long-term relaxation originates from the spin-changing nonforward collisions in the collision term, quadratic in differences of scattering lengths. Spinconserving forward scattering does not play a role in the dynamics; it only has a noticeable effect if spatial symmetry is broken by a magnetic field gradient, as in studies of spin waves $[28,35,46]$, which is not the case in our setup. The anticommutator in Eq. (4) leads to a mean-field-driven correction to the trapping potential that is, however, negligible in the experiments considered here.

The collision integral [Eq. (5)] enables us to determine whether our system is in the collisionless, hydrodynamic, or intermediate regime. The average collision time in the 3D setup is $\tau_{3 \mathrm{D}} \sim\left(4 \pi a^{2} n_{p} v\right)^{-1}$, with the relevant scattering length $a$, peak density $n_{p}$ and velocity $v=\max \left(v_{T}, v_{F}\right)$, where $v_{T}=\sqrt{k_{B} T / M}$ denotes the thermal velocity and $v_{F}=$ $\sqrt{2 E_{F} / M}$ the Fermi velocity. It ranges from approximately 10 to $50 \mathrm{~ms}$ for spin-conserving collisions and approximately 1 to $5 \mathrm{~s}$ in the spin-changing case. Compared to the average trapping frequency of $\bar{\omega}=\left(\omega_{x} \omega_{y} \omega_{z}\right)^{1 / 3} \approx 2 \pi \times 58 \mathrm{~Hz}$, we obtain values for $\bar{\omega} \tau_{3 \mathrm{D}}$ between 3.5 and 17 for the spin-conserving collisions and between 350 and 1700 in the spin-changing case. The lowest and highest values of $\bar{\omega} \tau_{3 \mathrm{D}}$ are reached for the lowest and highest densities shown in Fig. 5, respectively. Hence, we may approach the hydrodynamic regime, where the collision rate is larger than $\bar{\omega}$ and local equilibrium can be established. On the other hand, our system becomes almost collisionless regarding the spin-changing collisions. Generally, we are in an intermediate regime. In the $1 \mathrm{D}$ case, collision times $\tau_{1 \mathrm{D}} \sim$ $\left(n_{p} \omega_{y} \omega_{z} a^{2} / v\right)^{-1}$ are on the order of 1 and $110 \mathrm{~ms}$, respectively, meaning that $\omega \tau_{1 \mathrm{D}} \sim 0.6$ and $\omega \tau_{1 \mathrm{D}} \sim 60$, concerning spin-conserving and spin-changing collisions, respectively. Hence, with respect to the former, the system would be hydrodynamic. However, it is still in an intermediate regime regarding the redistribution of particles among the spin states driven by spin-changing collisions.

\section{DISSIPATIVE REDISTRIBUTION OF SPIN OCCUPATIONS}

In the following, we focus on the long-term spin relaxation shown in Figs. 2(b) and 3, while recent experiments have studied spin oscillations and their damping [36]. In order to restrict the dynamics to this process, we initially prepare a spin mixture consisting of only the spin states $m= \pm 1 / 2$ without coherences. In this case, the coherent oscillations driven by the commutator in Eq. (4) are absent and the Wigner function $W_{m n}$ remains diagonal at all times. In the following, we investigate theoretically and experimentally this spinrelaxation dynamics in 3D as well as $1 \mathrm{D}$ systems. For a direct comparison between theory and experiment, we realize a 1D system employing a deep 2D optical lattice, which confines the atoms into tight elongated tubes $[8,49]$, as described in Appendix A 1. As shown in Fig. 4(a), the system gradually occupies all spin states and evolves toward a state of almost equal spin populations on a time scale of milliseconds. As a key result, we can well reproduce the experimentally observed dynamics using the full 1D Boltzmann equation without free parameters.

For harmonically trapped 3D systems, where all trap frequencies are about equal, we derive the full 3D version of Eq. (4) as well [see Eq. (28) in Sec. IV]. However, numerical simulations of this equation are too demanding numerically. Nevertheless, the trap-induced motion of the particles is considerably faster than mean-field or relaxation dynamics, which averages the spatial dependence of the interaction via dynamically created long-range interactions. The Wigner function then approximately separates into a product $\quad W_{m n}(\boldsymbol{x}, \boldsymbol{p}, t) \approx M_{m n}(t) \cdot f_{0}(\boldsymbol{x}, \boldsymbol{p}) \quad[22,36,48]$. The spatial part is assumed to be time independent and given by the initial equilibrium distribution $f_{0}(\boldsymbol{x}, \boldsymbol{p})=$ $\left[\exp \left(\frac{1}{k_{B} T}\left[\frac{p^{2}}{2 M}+\frac{1}{2} M\left(\omega_{x}^{2} x^{2}+\omega_{y}^{2} y^{2}+\omega_{z}^{2} z^{2}\right)-\mu\right]\right)+1\right]^{-1}$. We substitute this expression into the 3D kinetic equation (28) with the appropriate collision term. Hence, for negligible 

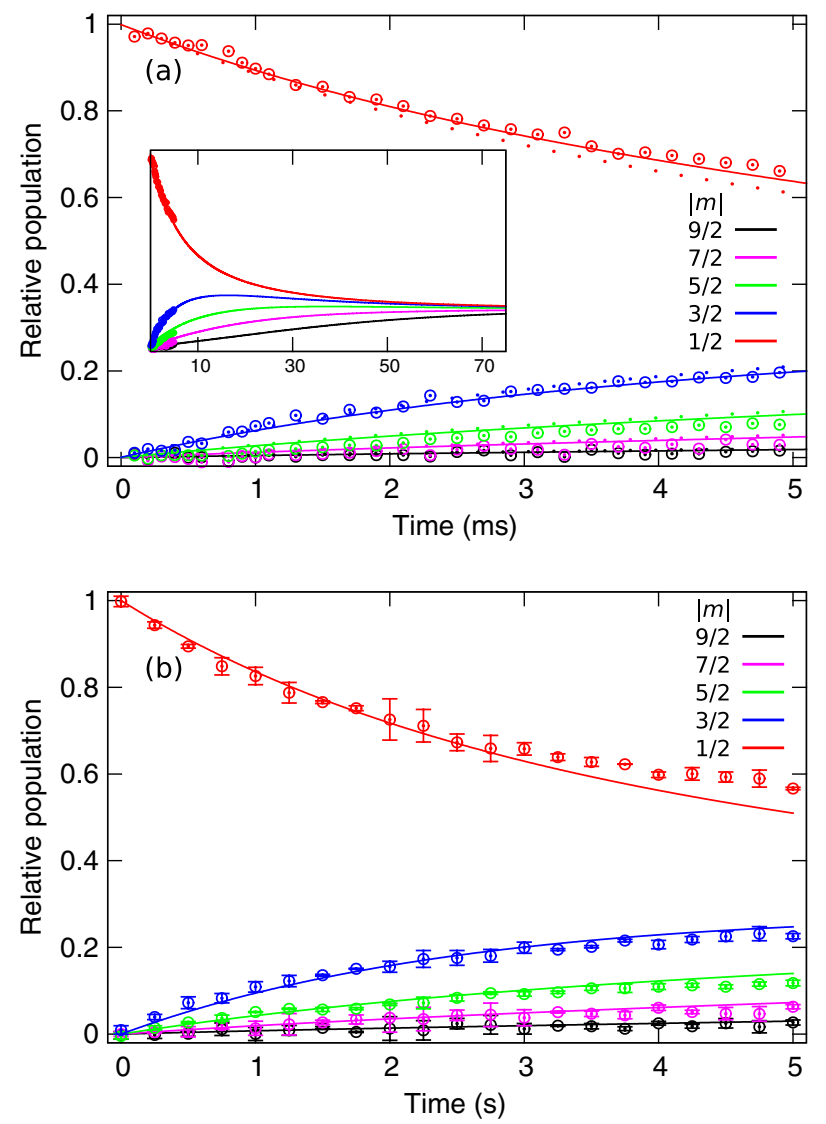

FIG. 4. Comparison of spin relaxation for $1 \mathrm{D}$ and 3D. The initial spin configuration is a mixture of $m= \pm 1 / 2$. (a) Experimental data in a $1 \mathrm{D}$ geometry (circles) compared to numerical results (lines) from the 1D Boltzmann equation (4) and (dots) from a 1D version of the single-mode approximation [Eq. (9)]. The axial trapping frequency is $\omega_{x}=2 \pi \times 84 \mathrm{~Hz}$, and radial frequencies are $\omega_{y, z}=2 \pi \times 47 \mathrm{kHz}$, particle number $N=100$ per tube at temperature $T / T_{F}=0.2$, and magnetic field $B=0.12 \mathrm{G}$. Inset: The system approaches a steady state for longer times. (b) Experimental data (circles) in a 3D configuration compared to calculations (lines) in the single-mode approximation [Eq. (9)] $\vec{\omega}=2 \pi \times(33,33,137) \mathrm{Hz}, N=1.3 \times 10^{5}$, and $T / T_{F}=0.15$ at $B=0.34 \mathrm{G}$.

magnetic fields, we find an equation for the matrix $M_{m n}(t)$ given by

$$
\frac{d}{d t} M_{m n}=-\lambda \sum_{a b c d} T_{m n}^{a b c d} M_{a c} M_{b d}
$$

where

$$
T_{m n}^{a b c d}=\frac{M}{4 \pi \hbar^{4}}\left(\tilde{U}_{m a b d}^{\prime} \delta_{n c}+\tilde{U}_{n c b d}^{\prime} \delta_{m a}-\sum_{l} U_{m a l b}^{\prime} U_{n c l d}^{\prime}\right)
$$

and

$$
\lambda=\frac{1}{N} \int d \boldsymbol{r} \int d \boldsymbol{p} \int d \boldsymbol{q}|\boldsymbol{q}| f_{0}(\boldsymbol{r}, \boldsymbol{p}) f_{0}(\boldsymbol{r}, \boldsymbol{p}-\boldsymbol{q}) .
$$

The 3D coupling constants are given by

$$
U_{a c b d}^{\prime}=\frac{4 \pi \hbar^{2}}{M} \sum_{S, M} a_{S}\langle a b \mid S M\rangle\langle S M \mid c d\rangle
$$

and

$$
\tilde{U}_{a c b d}^{\prime}=\frac{16 \pi^{2} \hbar^{4}}{M^{2}} \sum_{S, M} a_{S}^{2}\langle a b \mid S M\rangle\langle S M \mid c d\rangle .
$$

Note that in the 1D case, the single-mode approximation has a similar form given by $T_{m n}^{a b c d}=$ $\frac{M}{\hbar^{2}}\left(\tilde{U}_{m a b d} \delta_{n c}+\tilde{U}_{n c b d} \delta_{m a}-\sum_{l} U_{m a l b} U_{n c l d}\right)$.

For both single-mode approximations, the quadratic Zeeman shift has been neglected in the above equations, which are thus valid for small magnetic fields only (see Appendix $\mathrm{G}$ for full equations). In Fig. 4, we compare results from a single-mode approximation with experiments in a harmonically trapped Fermi gas yielding a surprisingly good agreement without free parameters. Note the qualitatively comparable behavior on different time scales of milliseconds for $1 \mathrm{D}$ and seconds for $3 \mathrm{D}$. On the contrary, the damping of the coherent spin oscillations visible in Figs. 2(a) and 3 is not described by this approach. The latter results from the assumption for the single-mode approximation that it completely neglects the multimode character of the fermionic many-body system and thus cannot account for spatial redistribution via lateral scattering events.

The high degree of control over all crucial parameters allows for a detailed investigation of the spin redistribution. To obtain further insight into the relaxation mechanisms, we measure the relaxation dynamics (as exemplarily shown in Fig. 4) for different densities while keeping $T / T_{F}$ constant. With higher density, the collision rate increases and the relaxation process accelerates, as shown in Fig. 5. The measured rates correspond to the redistribution of the initially populated components $m=$ $\pm 1 / 2$ into $m= \pm 3 / 2, \pm 5 / 2$ and are well reproduced using the single-mode approximation [Eq. (9)]. The rate of spin-changing collisions increases with increasing density, in accordance with the density dependence of the integral $\lambda$ [Eq. (10)].

As a second important parameter of the system, we investigate the influence of the magnetic field on the relaxation process. As the Zeeman energy of an atom pair changes during a spin-changing collision, a strong magnetic field suppresses this process by increasing the energy difference between the initial and final spin configurations. In Fig. 6(a), we depict the experimentally obtained populations of the spin components after $2 \mathrm{~s}$ 


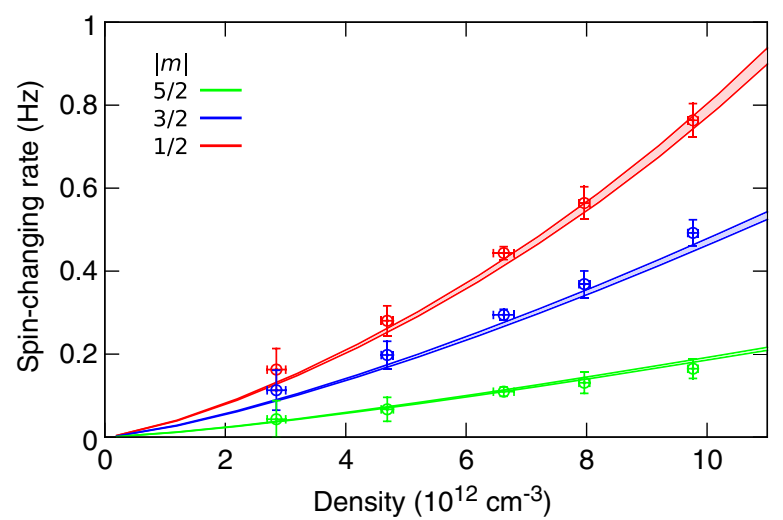

FIG. 5. Density dependence of the spin-relaxation rate in 3D, with an initial mixture of atoms in $m= \pm 1 / 2$. The spin-changing rate is obtained by fitting the solution of coupled rate equations to experimental (points) and theoretical (lines) data. Theoretical values are obtained using the single-mode approximation Eq. (9). The magnetic field is $B=0.11 \mathrm{G}$. We experimentally tune the density by changing the particle number, keeping the temperature constant at $T / T_{F}=0.26$.

as a function of the magnetic field strength (see Appendix A 2 for details) and compare them to singlemode [Fig. 6(a)] and 1D calculations [Fig. 6(b)] after $2 \mathrm{~ms}$. In both cases, the general behavior is very similar and shows a suppression of spin-changing collisions for large magnetic fields. Spin configurations with high values of $|m|$ are energetically significantly separated from the initially populated $m= \pm 1 / 2$ and are only occupied at very low field strengths. By changing the magnetic field, we can thus tune the magnitude of spin-changing collisions relative to the unaffected spinconserving collisions up to a complete suppression. Hence, one can regard the $m= \pm 1 / 2$ subsystem as a dissipative two-component Fermi gas with a tunable loss mechanism.

We have further investigated the time evolution of the temperature of this subsystem exposed to losses induced by these spin- and momentum-changing collisions. We compare two experiments: On the one hand, we perform an experiment at a high magnetic field $(B=7.6 \mathrm{G})$, where spin-changing collisions are suppressed. On the other hand, we perform a second experiment at a low magnetic field $(B=0.12 \mathrm{G})$ with strong spin relaxation.

In both cases, the system is in the hydrodynamic limit with respect to external degrees of freedom due to the comparatively large spin-conserving interactions. Hence, we make the assumption that at each time, the subsystems are close to an intermediate equilibrium state with a welldefined temperature. On the other hand, spin-changing collisions are at least 2 orders of magnitude weaker and very slowly change the particle number in each subsystem. This situation is reminiscent of prethermalization: First, a "prethermal" state is reached under the assumption of
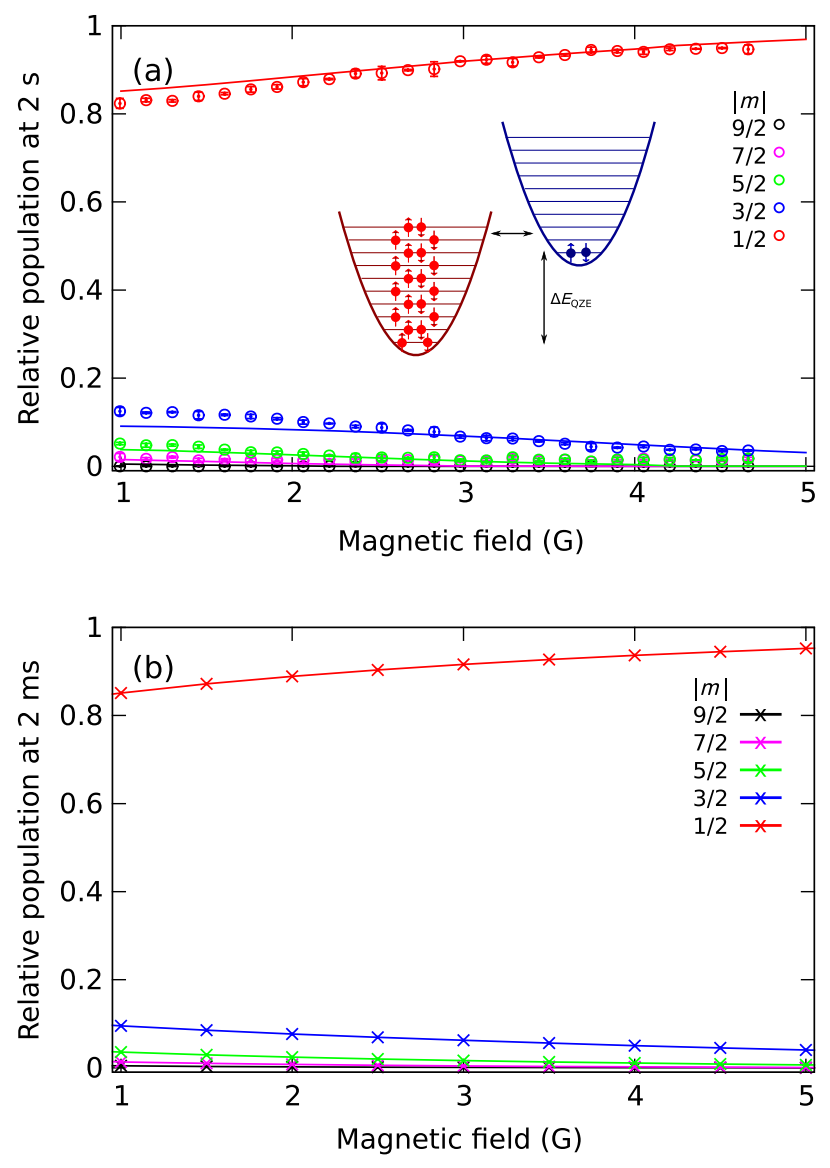

FIG. 6. Dependence of spin relaxation on magnetic field. (a) Experimental data, obtained from a 3D experiment (circles) and theoretical results from a single-mode approach (lines). Spin populations are measured after $2 \mathrm{~s}$. (b) Spin populations after $2 \mathrm{~ms}$, as obtained from full 1D simulations. The inset sketches how the interplay of the differential QZE and Fermi energy determines the probabilities for lateral spin-changing collisions.

conserved quantities that on a much longer time scale are actually not fully conserved due to a "slightly broken" symmetry, leading eventually to full thermalization [7]. Here, the role of the nearly conserved quantities is played by the occupation numbers of the ten spin states, which change only on a very long time scale. We measure the time evolution of the temperature of the initially populated $m=$ $\pm 1 / 2$ components and compare the temperatures for both cases described above. For large magnetic fields, we observe a small heating rate, which we mainly attribute to inelastic photon scattering. However, at low magnetic fields, the heating rate is significantly increased. In Fig. 7(a), we plot the temperature difference to extract the heating contributions solely generated by spin-changing collisions. This additional increase in temperature is due to hole creation in the Fermi sea [50] by scattering into the unoccupied spin states. We initially prepare a very cold two-component Fermi sea, with only a few unoccupied trap levels below the Fermi energy. Losses through 

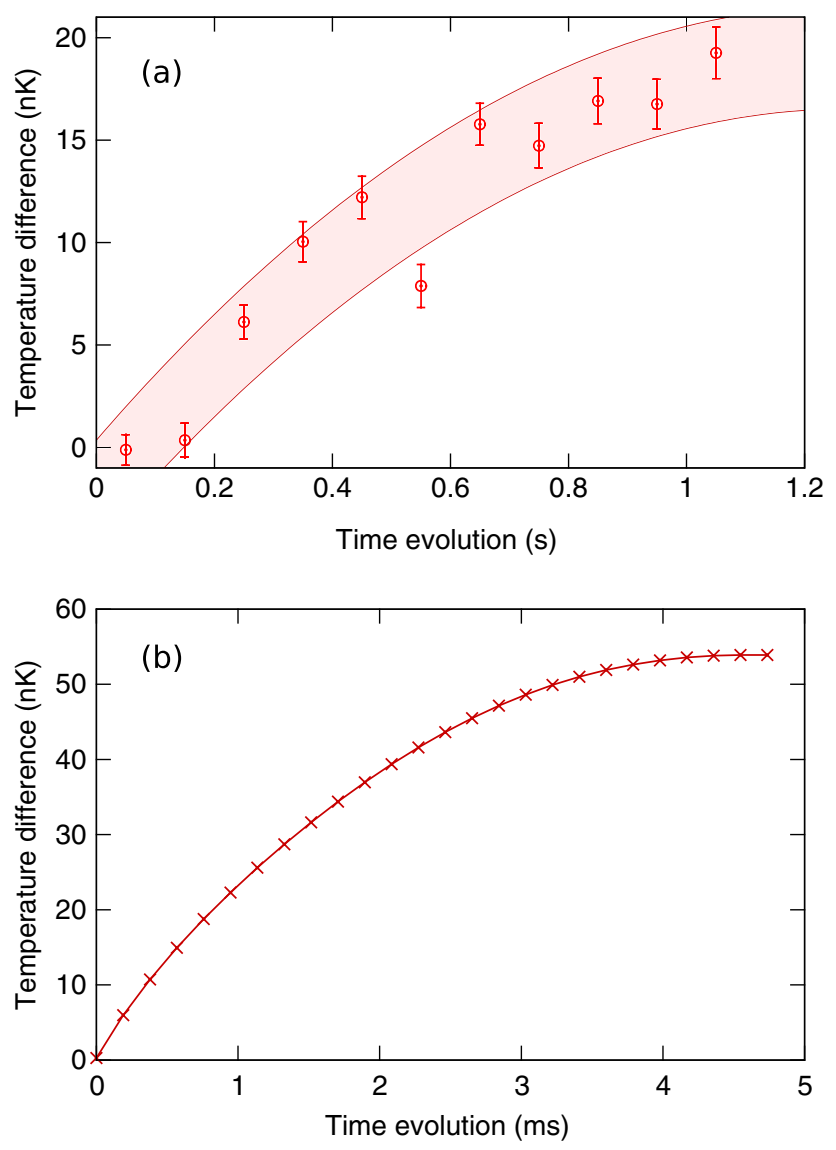

FIG. 7. Temperature increase due to spin redistribution. (a) Time evolution of the temperature difference between a closed (high magnetic field at $B=7.6 \mathrm{G}$ ) and a maximally open system (low magnetic field at $B=0.12 \mathrm{G}$ ). The shaded area serves as a guide to the eye. The particle number is $N=3.9 \times$ $10^{5}$ at an initial temperature $T=0.24 T_{F}=65 \mathrm{nK}$. (b) Results from a simulation of the $1 \mathrm{D}$ equation (4) at magnetic fields $B=$ $0.1 \mathrm{G}$ and $B=8 \mathrm{G}$. The method used to obtain the temperature is discussed in Appendix H.

spin-changing collisions "perforate" this Fermi sea with holes, such that the experimentally obtained temperature increases. Numerical simulations using the Boltzmann equation (4) confirm the experimentally observed heating induced by redistribution [see Fig. 7(b)].

\section{MICROSCOPIC DERIVATION OF A LARGE-SPIN BOLTZMANN EQUATION}

In this section, we describe the derivation of the $1 \mathrm{D}$ Boltzmann equation (4) in more detail. The reader not immediately interested in the details may skip this section and proceed directly to the conclusions. We follow previous work on the theoretical description of spin-polarized systems of $\mathrm{H}$ or He [43], called the Lhuillier-Laloë transport equation. We extend it to describe a 1D system with large spin, several scattering channels, and a quadratic Zeeman effect. We consider this approach to be suitable for our purpose for a couple of reasons. The entire equation is derived from a microscopic collisional approach, so the collision term is not based on phenomenological assumptions. We avoid the use of a relaxation-time approximation, widely used to describe damping in bosonic and fermionic systems [51-53], where the collision term is approximated by $I_{\text {coll }}[W]=\left(W_{\text {eq }}-W\right) / \tau$, with a relaxation time $\tau$. The reason is that for a multicomponent system, determining the equilibrium state $W_{\text {eq }}$ is very challenging. Also, due to the interplay of many different scattering lengths, we expect not one but many different relaxation times for each spin component. Our approach allows us to better understand the relaxation process itself, rather than merely its effect on other processes. Furthermore, from a technical point of view, our approach remains quadratic in the Wigner function, so it can be numerically simulated using the same standard techniques as the collisionless case $[28,47,48]$.

The idea behind the approach of Lhuillier and Laloë is to interpret the collision integral as the change rate of the state of a single particle $\hat{\rho} \rightarrow \hat{\rho}^{\prime}$ due to binary collisions

$$
I_{\text {coll }}=\frac{\hat{\rho}^{\prime}-\hat{\rho}}{\Delta t} .
$$

Here, $\Delta t$ is the elapsed time interval, which is short compared to any relevant macroscopic dynamics of the system but nevertheless is longer than the duration of a single collision, which is thus considered to be effectively instantaneous. This quantity will drop out and not appear in the final kinetic equation. Therefore, we treat collisions in the asymptotic limit, where they are described by the Heisenberg $S$-matrix. It relates the two-body density matrix of both scattering particles before a collision $\hat{\rho}(1,2)$ with the one after a collision $\hat{\rho}(1,2)^{\prime}$. Here, $(1,2)$ label the quantum numbers of particles 1 and 2 in first quantization. We obtain

$$
\hat{\rho}(1,2)^{\prime}=\hat{\mathcal{S}} \hat{\rho}(1,2) \hat{\mathcal{S}}^{\dagger} .
$$

In order to arrive at a single-particle description, we trace out particle 2 later. We next assume that particles involved in a collision are uncorrelated $\hat{\rho}(1,2)=\hat{\rho}(1) \otimes \hat{\rho}(2)$, both before and after the collision, an assumption justified for a system with a large number of particles. This assumption, in fact, corresponds to Boltzmann's original molecular chaos hypothesis (Stosszahlansatz). For the desired singleparticle density matrices before and after a collision, we obtain

$$
\begin{gathered}
\hat{\rho}(1)=\frac{1}{2} \operatorname{Tr}_{2}\left\{\left(\mathbb{1}-\mathcal{P}^{\mathrm{ex}}\right) \hat{\rho}(1) \otimes \hat{\rho}(2)\left(\mathbb{1}-\mathcal{P}^{\mathrm{ex}}\right)\right\}, \\
\hat{\rho}^{\prime}(1)=\frac{1}{2} \operatorname{Tr}_{2}\left\{\left(\mathbb{1}-\mathcal{P}^{\mathrm{ex}}\right) \hat{\mathcal{S}} \hat{\rho}(1) \otimes \hat{\rho}(2) \hat{\mathcal{S}}^{\dagger}\left(\mathbb{1}-\mathcal{P}^{\mathrm{ex}}\right)\right\},
\end{gathered}
$$


where we account for the indistinguishability of particles, with the operator $\mathcal{P}^{\text {ex }}$ exchanging the quantum numbers of particles 1 and 2. Because of fermionic statistics, it comes with a minus sign. This ansatz yields the following expression for the collision integral:

$$
\begin{aligned}
\hat{I}_{\text {coll }} \approx & \frac{1}{\Delta t} \operatorname{Tr}_{2}\left\{\frac { \mathbb { 1 } - \mathcal { P } ^ { \mathrm { ex } } } { \sqrt { 2 } } \left[\hat{\mathcal{S}} \hat{\rho}(1) \otimes \hat{\rho}(2) \hat{\mathcal{S}}^{\dagger}\right.\right. \\
& \left.-\hat{\rho}(1) \otimes \hat{\rho}(2)] \frac{\mathbb{1}-\mathcal{P}^{\mathrm{ex}}}{\sqrt{2}}\right\} .
\end{aligned}
$$

The $S$-matrix is related to the $T$-matrix via $\hat{\mathcal{S}}=\mathbb{1}-2 \pi i \hat{\mathcal{T}}$ such that Eq. (17) becomes

$$
\begin{aligned}
\hat{I}_{\text {coll }} \approx & \frac{2 \pi}{\Delta t} \operatorname{Tr}_{2}\left\{\frac { \mathbb { 1 } - \mathcal { P } ^ { \mathrm { ex } } } { \sqrt { 2 } } \left[i \hat{\mathcal{T}} \hat{\rho}(1) \otimes \hat{\rho}(2)-i \hat{\rho}(1) \otimes \hat{\rho}(2) \hat{\mathcal{T}}^{\dagger}\right.\right. \\
& \left.\left.+2 \pi \hat{\mathcal{T}} \hat{\rho}(1) \otimes \hat{\rho}(2) \hat{\mathcal{T}}^{\dagger}\right] \frac{\mathbb{1}-P^{\mathrm{ex}}}{\sqrt{2}}\right\} .
\end{aligned}
$$

This expression then has to be evaluated in the phase-space representation. Before performing the trace operation, we compute the two-body Wigner transform of the expression in braces in Eq. (18), which we demonstrate in detail in Appendix B, as well as the subsequent trace, shown in Appendix C. In the course of these calculations, we require the elements of the $S$-matrix. In the center-of-mass system, they are given by

$$
\begin{aligned}
\langle 1: & \left.k, a ; 2:-k, b|\hat{\mathcal{T}}| 1: k^{\prime}, c ; 2:-k^{\prime}, d\right\rangle \\
= & -2 \pi i \delta\left(\epsilon_{k}-\epsilon_{k^{\prime}}+Q_{a b c d}\right) \\
& \times\left\langle 1: a, 2: b\left|\hat{T}\left(k, k^{\prime}\right)\right| 1: c, 2: d\right\rangle,
\end{aligned}
$$

where $k, k^{\prime}$ denote the incoming and outgoing wave vectors of the particles and $m, n ; m^{\prime}, n^{\prime}$ denote the incoming and outgoing spins, respectively. The delta function assures energy conservation, $\epsilon_{k}=\hbar^{2} k^{2} / 2 \mu$ denotes kinetic energy with reduced mass $\mu=M / 2$, and $Q_{a b c d} \equiv Q\left(a^{2}+b^{2}-\right.$ $\left.c^{2}-d^{2}\right)$ denotes the shift in the quadratic Zeeman energy induced by a spin-changing collision. The on-shell $T$-matrix $\hat{T}\left(k, k^{\prime}\right)$ depends formally on the relative wave vectors $k, k^{\prime}$, but for our case of $s$-wave scattering, the dependence is only on the modulus. As they are related by energy conservation $\left|k^{\prime}\right|=\sqrt{k^{2}+Q_{a b c d}}$, the dependence is effectively only on $k$ or $k^{\prime}$. The QZE shift vanishes for spin-conserving collisions; hence, it is absent in the spin$1 / 2$ case and has the effect that scattering processes with a large $Q_{a b c d}$ are suppressed because the $T$-matrix decays approximately $1 /\left|k^{\prime}\right|$ for large $\left|k^{\prime}\right|$. For ${ }^{40} \mathrm{~K}$, the splitting is given by the quadratic part of the Breit-Rabi formula [54] $Q=-\left(2 \mu_{B}^{2}\left(g_{J}-g_{I}\right)^{2} B^{2}\right) / 9^{3} a_{\mathrm{HFS}}$, with the Bohr magneton $\mu_{B}$, nuclear and electronic $g$ factors $g_{I}$ and $g_{J}$, and hyperfine structure coefficient $a_{\mathrm{HFS}}$ [55].
To account for the spin-dependent interactions, we separate the $T$-matrix into channels of total spin $S$ and magnetization $M$ and obtain elements

$$
\begin{aligned}
& \left\langle 1: a, 2: b\left|\hat{T}\left(k, k^{\prime}\right)\right| 1: c, 2: d\right\rangle \equiv T_{a b c d}\left(k, k^{\prime}\right) \\
& \quad=\sum_{S M} T_{S}\left(k, k^{\prime}\right)\langle a b \mid S M\rangle\langle S M \mid c d\rangle .
\end{aligned}
$$

For a 1D system, the expression for a $T$-matrix in the channel with a coupling constant $g_{S}$ is

$$
T_{S}\left(k, k^{\prime}\right)=\frac{1}{2 \pi} \frac{i k^{\prime} \frac{2 \hbar^{2}}{M}}{1-i k^{\prime} \frac{2 \hbar^{2}}{M g_{S}}} .
$$

In each scattering channel, we perform a low-energy expansion in the coupling constant $g_{S} \rightarrow 0$ up to second order, to maintain the unitarity of the $S$-matrix. Since for the $1 \mathrm{D}$ case, the expansion in powers of $g_{S}$ is accompanied by factors of $\left(k^{\prime}\right)^{-1}$, we artificially create a singularity in the imaginary part of the $T$-matrix in this procedure. We remedy this problem by choosing a cutoff $\left|k^{\prime}\right|<M g_{S} / 2 \hbar^{2}$, which is the distance between 0 and the maximum of the imaginary part of the $T$-matrix (see Appendix D for more details). This step is unnecessary in the $3 \mathrm{D}$ case discussed by Lhuillier and Laloë $[43,44,46]$. The result is then given by

$$
T_{S}\left(k, k^{\prime}\right) \approx \frac{g_{S}}{2 \pi}- \begin{cases}0 & \text { if }\left|k^{\prime}\right|<\frac{M g_{S}}{2 \hbar^{2}} \\ \frac{i M g_{S}^{2}}{4 \pi \hbar^{2} k^{\prime}}+\cdots & \text { if }\left|k^{\prime}\right| \geq \frac{M g_{S}}{2 \hbar^{2}}\end{cases}
$$

or, respectively,

$T_{a b c d}\left(k, k^{\prime}\right) \approx \frac{U_{a c b d}}{2 \pi}- \begin{cases}0 & \text { if }\left|k^{\prime}\right|<\frac{M U_{a c b d}}{2 \hbar^{2}} \\ \frac{i M \tilde{U}_{a c b d}}{4 \pi \hbar^{2} k^{\prime}}+\cdots & \text { if }\left|k^{\prime}\right| \geq \frac{M U_{a c b d}}{2 \hbar^{2}} .\end{cases}$

The leading terms linear in $g_{S}$ correspond to forward scattering, the quadratic terms to backward (lateral in higher dimensions) scattering processes.

The expansion of the $T$-matrix is performed in addition to a semiclassical gradient expansion of the Wigner function (see Appendix E for details) to first order in the linear terms and to zero order in the quadratic expressions. During this procedure, we encounter squares of delta functions whose interpretation is described in Appendix F. Finally, we obtain a collisional integral, consisting of three parts 


$$
I_{m n}^{\mathrm{coll}}(x, p)=I_{m n}^{\mathrm{MF}}(x, p)+I_{m n}^{T}(x, p)+I_{m n}^{T^{2}}(x, p),
$$

where $I^{\mathrm{MF}}$ is linear in $a_{S}$ and contains the forwardscattering part of collisions leading to phase shifts

$$
\begin{aligned}
I_{m n}^{\mathrm{MF}}(x, p)= & -\frac{i}{\hbar} \sum_{l}\left[V_{n l}^{\mathrm{MF}}(x) W_{m l}(x, p)-W_{l m}(x, p) V_{l n}^{\mathrm{MF}}(x)\right] \\
& +\frac{1}{2} \sum_{l}\left\{\partial_{x} V_{n l}^{\mathrm{MF}}(x) \partial_{p} W_{m l}(x, p)\right. \\
& \left.+\partial_{p} W_{l m}(x, p) \partial_{x} V_{l n}^{\mathrm{MF}}(x)\right\}
\end{aligned}
$$

It coincides with the interaction term obtained from the treatment of the same problem on a simpler mean-field level $[28,48]$. Because of its effect as a nonlinear modification of the trap and magnetic field, in Eq. (4), we have separated this term from the collisional integral and added it to the free motion of the particles in the external fields. The quadratic terms that form Eq. (5) contain backward scattering, including momentum exchange between particles. They appear as dissipation on the single-particle level and are given by

$I_{m n}^{T}(x, p)=-\frac{M}{\hbar^{2}} \sum_{a b l}\left[\int_{q^{2}>\epsilon_{1}} d q \frac{\tilde{U}_{\text {malb }}}{\sqrt{q^{2}+\Delta_{\text {mlab }}}} W_{a n}(x, p) W_{b l}(x, p-q)+\int_{q^{2}>\epsilon_{2}} d q \frac{\tilde{U}_{\text {nalb }}}{\sqrt{q^{2}+\Delta_{n l a b}}} W_{m a}(x, p) W_{l b}(x, p-q)\right]$,

$I_{m n}^{T^{2}}(x, p)=\frac{M}{\hbar^{2}} \sum_{a b c d l} \int_{q^{2}>\epsilon_{3}} d q \frac{U_{\text {malb }} U_{n c l d}}{\sqrt{q^{2}+\Delta_{\text {mnlabcd }}}} W_{a c}\left(x, p-\frac{1}{2}\left(q-\sqrt{q^{2}+\Delta_{\text {mnlabcd }}}\right)\right) W_{b d}\left(x, p-\frac{1}{2}\left(q+\sqrt{q^{2}+\Delta_{\text {mnlabcd }}}\right)\right)$.

The integration domain cutoffs around $q=0$, coming from Eq. (23), are given by $\epsilon_{1}=\left(M U_{\text {malb }} / \hbar\right)-\Delta_{\text {mlab }}$, $\epsilon_{2}=\left(M U_{\text {nalb }} / \hbar\right)-\Delta_{\text {nlab }}$, and $\epsilon_{3}=M U_{\text {nalb }} / 2 \hbar\left(U_{\text {malb }}+U_{\text {ncld }}\right)-\Delta_{\text {mnlabcd }}$. The corresponding result for a full 3D calculation (see Ref. [46] for the case of spin 1/2) reads

$$
\begin{aligned}
& \frac{d}{d t} W_{m n}(\boldsymbol{r}, \boldsymbol{p})+\left[\frac{\boldsymbol{p}}{M} \cdot \nabla_{r}-M\left(\omega_{x}^{2} x, \omega_{y}^{2} y, \omega_{z}^{2} z\right) \cdot \nabla_{p}+\frac{i Q}{\hbar}\left(n^{2}-m^{2}\right)\right] W_{m n}(\boldsymbol{r}, \boldsymbol{p})+\frac{i}{\hbar} \sum_{l}\left[V_{n l}^{\mathrm{MF}}(\boldsymbol{r}) W_{m l}(\boldsymbol{r}, \boldsymbol{p})-W_{l m}(\boldsymbol{r}, \boldsymbol{p}) V_{l n}^{\mathrm{MF}}(\boldsymbol{r})\right] \\
& \quad-\frac{1}{2} \sum_{l}\left\{\nabla_{r} V_{n l}^{\mathrm{MF}}(\boldsymbol{r}) \cdot \nabla_{p} W_{m l}(\boldsymbol{r}, \boldsymbol{p})+\nabla_{p} W_{l m}(\boldsymbol{r}, \boldsymbol{p}) \cdot \nabla_{r} V_{l n}^{\mathrm{MF}}(\boldsymbol{r})\right\}=I_{m n}^{\mathrm{coll}}(\boldsymbol{r}, \boldsymbol{p}) .
\end{aligned}
$$

The mean-field potential is given by $V_{m n}^{\mathrm{MF}}(\boldsymbol{r})=2 \int d \boldsymbol{p} \sum_{a b} U_{m n a b}^{\prime} W_{a b}(\boldsymbol{r}, \boldsymbol{p})$, and the collision term reads

$$
\begin{aligned}
I_{m n}^{\mathrm{coll}}(\boldsymbol{r}, \boldsymbol{p})= & -\frac{M}{4 \pi \hbar^{4}} \int d \boldsymbol{q}\left\{\sum_{a b c}\left[\sqrt{q^{2}+\Delta_{m c a b}} \tilde{U}_{m a c b}^{\prime} W_{a n}(\boldsymbol{r}, \boldsymbol{p}) W_{b c}(\boldsymbol{r}, \boldsymbol{p}-\boldsymbol{q})+\sqrt{q^{2}+\Delta_{a b n c}} \tilde{U}_{a n b c}^{\prime} W_{m a}(\boldsymbol{r}, \boldsymbol{p}) W_{c b}(\boldsymbol{r}, \boldsymbol{p}-\boldsymbol{q})\right]\right. \\
& \left.-\frac{1}{2 \pi} \int d \Omega \sum_{a b c d l} \sqrt{q^{2}+\Delta_{\text {mnlabcd }}} U_{m a l b}^{\prime} U_{n c l d}^{\prime} W_{a c}\left(\boldsymbol{r}, \boldsymbol{p}-\frac{1}{2}\left(\boldsymbol{q}-\boldsymbol{p}^{\prime}\right)\right) W_{b d}\left(\boldsymbol{r}, \boldsymbol{p}-\frac{1}{2}\left(\boldsymbol{q}+\boldsymbol{p}^{\prime}\right)\right)\right\}
\end{aligned}
$$

where $\boldsymbol{p}^{\prime}=\boldsymbol{e}_{\Omega} \sqrt{q^{2}+\Delta_{\text {mnlabcd }}}$ and $\boldsymbol{e}_{\Omega}$ denotes the unit vector corresponding to the solid angle $d \Omega$.

A physical interpretation of this expression can be obtained by looking at the origin of the individual terms. The upper two lines of Eq. (29) originate from the second order of the expansion of the $T$-matrix [Eq. (23)], describing the intensity shift in the forwardscattered wave $[43,46]$, while the first order only describes a phase shift and appears in the mean-field terms in Eq. (28). The bottom line of Eq. (29) contains all lateral scattering processes, hence the explicit angular dependence. In our formalism, the coupling constants $U$ and $\tilde{U}$ include all particle indistinguishability and exchange contributions, which are discussed in greater detail in Ref. [43].

\section{CONCLUSION}

We have presented a novel approach to study relaxation dynamics in a closed quantum system, exploiting the unique properties of a large-spin Fermi sea. For this system, we have derived a multicomponent kinetic equation without phenomenological assumptions or prior knowledge of the equilibrium state. As a key result, we find that this approach is well suited for the quantitative description of weakly interacting fermionic many-body systems with 
large spin. Both the comparison of numerical simulations with full spatial resolution to a $1 \mathrm{D}$ experiment as well as the comparison of a simplified single-mode approximation to a 3D experiment yield a very good agreement without free parameters. We identify different collisional processes on different time scales and identify spin relaxation as the slowest dynamical process of the system. A variation of the density and the geometry of the system changes the respective spin-relaxation rates by several orders of magnitude, ranging from a few milliseconds to several seconds. By tuning the magnetic field, we can precisely control the coupling strengths of individual collision channels, allowing us to tune the character of a subsystem of two spin components within the large-spin Fermi sea continuously from an open to a closed system. The spin relaxation manifests itself in a perforation of the Fermi sea accompanied with a temperature increase.

Our results broaden the understanding of many-body relaxation dynamics. In particular, the fermionic character of the system underlines its model character for various systems in nature. The possibility to individually monitor different spin components allows us to employ the largespin Fermi sea for novel studies of decoherence and relaxation processes in quantum many-body systems. Furthermore, spin-relaxation dynamics might play an important role for proposed fermionic large-spin phenomena, e.g., quantum-chromodynamic-like color superfluidity or large-spin texture formation.

\section{ACKNOWLEDGMENTS}

We acknowledge fruitful discussions with Jannes Heinze and Ludwig Mathey. This work has been funded by the Spanish Ministerio de Ciencia e Innovación (FIS 200800784, AAII-Hubbard, FPI Fellowship); ERC Grants QUAGATUA, OSYRIS, and EU IP SIQS; and Deutsche Forschungsgemeinschaft (DFG) Collaborate Research Center SFB 925 and DFG Excellence Cluster: The Hamburg Centre for Ultrafast Imaging-Structure, Dynamics, and Control of Matter on the Atomic Scale. U.E. and J. S. K. contributed equally to this work.

\section{APPENDIX A: EXPERIMENTAL DETAILS}

\section{Preparation}

We sympathetically cool spin-polarized ${ }^{40} \mathrm{~K}$ atoms in the state $|F=9 / 2, m=9 / 2\rangle$ down to a temperature of typically $0.1 T_{F}$ in a magnetic trap, using bosonic ${ }^{87} \mathrm{Rb}$ as a buffer gas. Subsequently, we transfer the atoms into a crossed circularelliptical optical dipole trap operated at a wavelength of $\lambda=812 \mathrm{~nm}$. Using radio-frequency (rf) pulses and $\mathrm{rf}$ sweeps, we create a spin mixture that we evaporate to quantum degeneracy by lowering the power of the dipole trap exponentially in $2 \mathrm{~s}$. We typically realize samples with particle numbers of the order of $N \sim 10^{5}$ at temperatures of $T=0.1 T_{F}-0.2 T_{F}$. After the evaporation, we compress the trap again to avoid particle loss during the experiments, realizing typical trapping frequencies of $\vec{\omega}=$ $2 \pi \times(33,33,137) \mathrm{Hz}$. By varying the evaporation sequence and including additional waiting times, we can control the initial temperature and particle number independently in the same trap geometry. Thereby, we can modify the density while keeping $T / T_{F}$ approximately constant. Typically, a balanced mixture of atoms in spin states $m= \pm 1 / 2$ is used as the initial state throughout this paper. To study the spinchanging dynamics, we switch the magnetic field to low values. In Fig. 2, in addition, a coherent superposition of several spin states is prepared by applying subsequently a rf pulse at low magnetic field corresponding to a spin rotation of $\theta=0.44$ (see Ref. [36] for more details). The 1D configuration used in Fig. 4(a) is realized by adiabatically ramping up a 2D optical square lattice over $150 \mathrm{~ms}$. The lattice is created by two orthogonal retroreflected laser beams at wavelength $\lambda=1030 \mathrm{~nm}$ with a $1 / e^{2}$ radius of $200 \mu \mathrm{m}$ detuned with respect to each other by several tens of megahertz. The lattice depth is $25 E_{\text {recoil }}$, with $E_{\text {recoil }}=\hbar^{2} k_{L}^{2} / 2 M$, where $k_{L}=2 \pi / \lambda$. This optical potential creates an array of $1 \mathrm{D}$ tubes, where a single tube can be described as a harmonically trapped system with frequencies $\omega_{x}=2 \pi \times 84 \mathrm{~Hz}$ and $\omega_{y, z}=2 \pi \times$ $47 \mathrm{kHz}$. With a particle number of $N \approx 100$ and $E_{F}=$ $2 \pi \hbar \times 37 \mathrm{kHz}$, the radial trapping frequencies fulfill $\hbar \omega_{y, z}>E_{F}$, and at a temperature $k_{B} T=0.2 E_{F}$, we can neglect a possible population of excited radial modes; hence, we create a true $1 \mathrm{D}$ system. The extension of the radial ground state is around 1378 Bohr radii and thus 1 order of magnitude larger than the scattering lengths [33]. We thus neglect the possibility of a confinement-induced resonance [56] and use the effective coupling constants $g_{S}^{1 \mathrm{D}}=2 \hbar \sqrt{\omega_{y} \omega_{z}} a_{S}$.

\section{Measurement}

The relative populations of spin components are measured as follows: We release the atoms from the trap in an inhomogeneous magnetic field to separate the spin components during a time-of-flight expansion of typically $18.5 \mathrm{~ms}$. We count the number of atoms in each spin component with resonant absorption imaging. For comparison, we measure the total number of particles as well as the temperature independently without the Stern-Gerlach field to avoid distortions of the particle cloud during the time of flight. The numbers given in this paper correspond to the initial temperature and particle number. In Fig. 7(a), in order to extract the change in temperature over time, we determine the temperature only in one spin component, circumventing deviations associated with the imbalance of the spin mixture. For instance, to measure the temperature in $m=1 / 2$, we apply a sequence of linearly polarized microwave pulses with a duration of $50 \mu$ s to transfer all significantly occupied spin components $m \neq 1 / 2$ into the $F=7 / 2$ hyperfine manifold of ${ }^{40} \mathrm{~K}$. In the other hyperfine manifold, the atoms are not resonant with the detection light and are thus obscured during the absorption imaging process. 


\section{APPENDIX B: TWO-BODY WIGNER TRANSFORM}

Because the $T$-matrix depends only on the relative wave vectors, we evaluate Eq. (18) in the center-of-mass frame. We introduce the notation

$$
R=\frac{1}{2}\left(x_{1}+x_{2}\right), \quad r=x_{1}-x_{2}, \quad P=p_{1}+p_{2}, \quad p=\frac{1}{2}\left(p_{1}-p_{2}\right)
$$

to denote the center of mass and relative positions and momenta versus the coordinates of particles 1 and 2 denoted by subscripts. We denote by $W^{\left(T, T^{2}\right)}$ the two-body Wigner transform of the part of Eq. (18) linear in the $T$-matrix $2 \pi i \frac{\mathbb{1}-\mathcal{P}^{\text {ex }}}{\sqrt{2}}\left[\hat{\mathcal{T}} \hat{\rho}(1) \otimes \hat{\rho}(2)-\hat{\rho}(1) \otimes \hat{\rho}(2) \hat{\mathcal{T}}^{\dagger}\right] \frac{\mathbb{1}-\mathcal{P}^{\text {ex }}}{\sqrt{2}}$ and the quadratic part $4 \pi^{2} \frac{\mathbb{1}-\mathcal{P}^{\text {ex }}}{\sqrt{2}}\left[\hat{\mathcal{T}} \hat{\rho}(1) \otimes \hat{\rho}(2) \hat{\mathcal{T}}^{\dagger}\right] \frac{\mathbb{1}-\mathcal{P}^{\text {ex }}}{\sqrt{2}}$, respectively. We obtain

$$
\begin{gathered}
W_{i j m n}^{T}(r, R, p, P)=\frac{-i}{2 \pi \hbar^{2}} \int d K \int d \kappa e^{i K R} e^{i \kappa r}\left\langle K_{+}, k_{+}, i, m\left|\frac{\mathbb{1}-\mathcal{P}^{\mathrm{ex}}}{\sqrt{2}} \hat{\mathcal{T}} \hat{\rho}(1) \otimes \hat{\rho}(2) \frac{\mathbb{1}-\mathcal{P}^{\mathrm{ex}}}{\sqrt{2}}\right| K_{-}, k_{-}, j, n\right\rangle+\text { H.c. } \\
W_{i j m n}^{T^{2}}(r, R, p, P)=\frac{1}{\hbar^{2}} \int d K \int d \kappa e^{i K R} e^{i \kappa r}\left\langle K_{+}, k_{+}, i, m\left|\frac{\mathbb{1}-\mathcal{P}^{\mathrm{ex}}}{\sqrt{2}} \hat{\mathcal{T}} \hat{\rho}(1) \otimes \hat{\rho}(2) \hat{\mathcal{T}}^{\dagger} \frac{\mathbb{1}-\mathcal{P}^{\mathrm{ex}}}{\sqrt{2}}\right| K_{-}, k_{-}, j, n\right\rangle
\end{gathered}
$$

where we introduce the wave vectors $K_{ \pm}=\frac{P}{\hbar} \pm \frac{K}{2}$ and $k_{ \pm}=\frac{p}{\hbar} \pm \frac{\kappa}{2}$. We insert two complete bases $\int d K_{1} \int d k_{1} \sum_{a b}\left|K_{1}, k_{1}, a, b\right\rangle\left\langle K_{1}, k_{1}, a, b\right|$ and $\int d K_{2} \int d k_{2} \sum_{c d}\left|K_{2}, k_{2}, c, d\right\rangle\left\langle K_{2}, k_{2}, c, d\right|$ to the left and right of the tensor product of density matrices. The dependence of the $T$-matrix on the relative wave vector only makes the integration over $K_{1,2}$ trivial. We substitute from Eq. (19) the expression

$$
\left\langle K_{1}, k_{1}, a, b|\hat{\mathcal{T}}| K_{2}, k_{2}, c, d\right\rangle=\delta\left(\epsilon_{k_{1}}-\epsilon_{k_{2}}+Q_{a b c d}\right) T_{a b c d}\left(k_{1}, k_{2}\right)
$$

for the elements of the $T$-matrix into the above expressions and obtain

$$
\begin{aligned}
W_{i j m n}^{T}(r, R, p, P)= & \frac{-i}{2 \pi \hbar^{2}} \int d K \int d \kappa \int d k_{1} \int d k_{2} e^{i K R} e^{i \kappa r} \sum_{a b c d}\left\langle K_{+}, k_{+}, i, m\left|\frac{\mathbb{1}-\mathcal{P}^{\mathrm{ex}}}{\sqrt{2}} \hat{\mathcal{T}}\right| K_{+}, k_{1}, a, b\right\rangle \\
& \times\left\langle K_{+}, k_{1}, a, b|\hat{\rho}(1) \otimes \hat{\rho}(2)| K_{-}, k_{2}, c, d\right\rangle\left\langle K_{-}, k_{2}, c, d\left|\frac{\mathbb{1}-\mathcal{P}^{\mathrm{ex}}}{\sqrt{2}}\right| K_{-}, k_{-}, j, n\right\rangle+\text { H.c. } \\
= & \frac{-i}{4 \pi \hbar^{2}} \int d K \int d \kappa \int d k_{1} \int d k_{2} e^{i K R} e^{i \kappa r} \sum_{a b c d} \delta\left(\epsilon_{k_{+}}-\epsilon_{k_{1}}+Q_{i m a b}\right)\left[\delta\left(k_{2}-k_{-}\right) \delta_{c j} \delta_{d n}-\delta\left(k_{2}+k_{-}\right) \delta_{n c} \delta_{j d}\right] \\
& \times\left[T_{\text {imab }}\left(k_{+}, k_{1}\right)-T_{\text {miab }}\left(-k_{+}, k_{1}\right)\right]\left\langle K_{+}, k_{1}, a, b|\hat{\rho}(1) \otimes \hat{\rho}(2)| K_{-}, k_{2}, c, d\right\rangle+\text { H.c. }
\end{aligned}
$$

for the linear term and

$$
\begin{aligned}
W_{i j m n}^{T^{2}}(r, R, p, P)= & \frac{1}{2 \hbar^{2}} \int d K \int d \kappa \int d k_{1} \int d k_{2} e^{i K R} e^{i \kappa r} \sum_{a b c d} \delta\left(\epsilon_{k_{+}}-\epsilon_{k_{1}}+Q_{i m a b}\right) \delta\left(\epsilon_{k_{2}}-\epsilon_{k_{-}}+Q_{c d j n}\right) \\
& \times\left[T_{i m a b}\left(k_{+}, k_{1}\right)-T_{\text {miab }}\left(-k_{+}, k_{1}\right)\right]\left[T_{j n c d}^{*}\left(k_{-}, k_{2}\right)-T_{n j c d}^{*}\left(-k_{-}, k_{2}\right)\right]\left\langle K_{+}, k_{1}, a, b|\hat{\rho}(1) \otimes \hat{\rho}(2)| K_{-}, k_{2}, c, d\right\rangle
\end{aligned}
$$

for the term quadratic in the $T$-matrix. The elements of the tensor product of density matrices are obtained from the Wigner functions by an inverse Wigner transform

$$
\begin{aligned}
\left\langle K_{+}, k_{1}, a, b\right| \hat{\rho}(1) \otimes & \hat{\rho}(2)\left|K_{-}, k_{2}, c, d\right\rangle=\hbar^{2} \int d R^{\prime} \int d r^{\prime} e^{-i K R^{\prime}} e^{i\left(k_{2}-k_{1}\right) r^{\prime}} \\
& \times W_{a c}\left(R^{\prime}+\frac{r^{\prime}}{2}, \frac{P+\hbar k_{1}+\hbar k_{2}}{2}\right) W_{b d}\left(R^{\prime}-\frac{r^{\prime}}{2}, \frac{P-\hbar k_{1}-k_{2}}{2}\right),
\end{aligned}
$$

and we substitute this expression into the collision term. We substitute this expression into the collision term, which yields a delta function $\int d K e^{i K\left(R-R^{\prime}\right)}=2 \pi \delta\left(R-R^{\prime}\right)$, and after carrying out the integration over $K$ and $R^{\prime}$, we obtain 


$$
\begin{aligned}
W_{i j m n}^{T}(r, R, p, P)= & \frac{-i}{2} \int d \kappa \int d k_{1} \int d k_{2} \int d r^{\prime} e^{i \kappa r} e^{i\left(k_{2}-k_{1}\right) r^{\prime}} \sum_{a b c d}\left[\delta\left(k_{2}-k_{-}\right) \delta_{c j} \delta_{d n}-\delta\left(k_{2}+k_{-}\right) \delta_{n c} \delta_{j d}\right] \\
& \times \delta\left(\epsilon_{k_{+}}-\epsilon_{k_{1}}+Q_{i m a b}\right)\left[T_{i m a b}\left(k_{+}, k_{1}\right)-T_{m i a b}\left(-k_{+}, k_{1}\right)\right] W_{a c}\left(R+\frac{r^{\prime}}{2}, \frac{P+\hbar k_{1}+\hbar k_{2}}{2}\right) \\
& \times W_{b d}\left(R-\frac{r^{\prime}}{2}, \frac{P-\hbar k_{1}-\hbar k_{2}}{2}\right)+\text { H.c. }
\end{aligned}
$$

and

$$
\begin{aligned}
W_{i j m n}^{T^{2}}(r, R, p, P)= & \pi \int d \kappa \int d k_{1} \int d k_{2} \int d r^{\prime} e^{i \kappa r} e^{i\left(k_{2}-k_{1}\right) r^{\prime}} \sum_{a b c d} \delta\left(\epsilon_{k_{+}}-\epsilon_{k_{1}}+Q_{i m a b}\right) \delta\left(\epsilon_{k_{2}}-\epsilon_{k_{-}}+Q_{c d j n}\right) \\
& \times\left[T_{\text {imab }}\left(k_{+}, k_{1}\right)-T_{\text {miab }}\left(-k_{+}, k_{1}\right)\right]\left[T_{j n c d}^{*}\left(k_{-}, k_{2}\right)-T_{\text {lncd }}^{*}\left(-k_{-}, k_{2}\right)\right] W_{a c}\left(R+\frac{r^{\prime}}{2}, \frac{P+\hbar k_{1}+\hbar k_{2}}{2}\right) \\
& \times W_{b d}\left(R-\frac{r^{\prime}}{2}, \frac{P-\hbar k_{1}-\hbar k_{2}}{2}\right) .
\end{aligned}
$$

\section{APPENDIX C: TRACE OVER SECOND PARTICLE}

In order to trace out particle 2 as described in Eq. (18), we return from the center-of-mass frame to the lab frame by substituting Eqs. (B1) back into Eq. (B9). The trace over particle 2 means performing the operation $I_{i j}^{\left(T, T^{2}\right)}\left(x_{1}, p_{1}\right)=$ $\frac{1}{\Delta t} \int d x_{2} \int d p_{2} \sum_{m n} \delta_{m n} W_{i j m n}^{\left(T, T^{2}\right)}(r, R, p, P)$ on each term. Introducing the notations $q=2 \hbar k, p_{1}^{\prime}=p_{1}-\left(q-\hbar\left(k_{1}+\right.\right.$ $\left.\left.k_{1}\right) / 2\right)$, and $p_{2}^{\prime}=p_{1}-\left(q+\hbar\left(k_{1}+k_{2}\right) / 2\right)$, we arrive at the following expressions for the collision term:

$$
\begin{aligned}
I_{i j}^{T}\left(x_{1}, p_{1}\right)= & \frac{-i}{2 \Delta t} \int d \kappa \int d k_{1} \int d k_{2} \int d r^{\prime} \int d r \int d q e^{i \kappa r} e^{i\left(k_{2}-k_{1}\right) r^{\prime}} \sum_{a b c d l} \delta\left(\epsilon_{k_{+}}-\epsilon_{k_{1}}+Q_{i l a b}\right)\left[\delta\left(k_{2}-k_{-}\right) \delta_{c j} \delta_{d l}\right. \\
& \left.-\delta\left(k_{2}+k_{-}\right) \delta_{l c} \delta_{j d}\right]\left[T_{i l a b}\left(k_{+}, k_{1}\right)-T_{l i a b}\left(-k_{+}, k_{1}\right)\right] W_{a c}\left(x_{1}-\frac{r-r^{\prime}}{2}, p_{1}^{\prime}\right) W_{b d}\left(x_{1}-\frac{r+r^{\prime}}{2}, p_{2}^{\prime}\right)+\text { H.c. }
\end{aligned}
$$

and

$$
\begin{aligned}
I_{i j}^{T^{2}}\left(x_{1}, p_{1}\right)= & \frac{\pi}{\Delta t} \int d \kappa \int d k_{1} \int d k_{2} \int d r^{\prime} \int d r \int d q e^{i \kappa r} e^{i\left(k_{2}-k_{1}\right) r^{\prime}} \sum_{a b c d l} \delta\left(\epsilon_{k_{+}}-\epsilon_{k_{1}}+Q_{i l a b}\right) \delta\left(\epsilon_{k_{2}}-\epsilon_{k_{-}}\right. \\
& \left.+Q_{c d j l}\right)\left[T_{i l a b}\left(k_{+}, k_{1}\right)-T_{l i a b}\left(-k_{+}, k_{1}\right)\right]\left[T_{j l c d}^{*}\left(k_{-}, k_{2}\right)\right. \\
& \left.-T_{l j c d}^{*}\left(-k_{-}, k_{2}\right)\right] W_{a c}\left(x_{1}-\frac{r-r^{\prime}}{2}, p_{1}^{\prime}\right) W_{b d}\left(x_{1}-\frac{r+r^{\prime}}{2}, p_{2}^{\prime}\right) .
\end{aligned}
$$

\section{APPENDIX D: ONE-DIMENSIONAL LARGE-SPIN $T$-MATRIX}

In the one-dimensional two-body scattering problem in the center-of-mass frame with Hamiltonian $H=$ $\left(-\hbar^{2} / 2 \mu\right)\left(d^{2} / d x^{2}\right)+g_{S} \delta(x)$, the wave function is $\psi(x)=e^{i k x}+f_{k^{\prime}} e^{i k^{\prime}|x|}$, from which follows $f_{k^{\prime}}=-1 /$ $\left(1-i \hbar^{2} k^{\prime} / \mu g_{S}\right)$ for the scattering amplitude. The scattered wave function $\psi_{\mathrm{sc}}\left(k^{\prime}\right)=f_{k^{\prime}} e^{i k^{\prime}|x|}$ and the $T$-matrix are related through the Green's function $\psi_{\mathrm{sc}}\left(k^{\prime}\right)=$ $G\left(k, k^{\prime}\right) T\left(k^{\prime}, k\right)$, which in 1D is given by

$$
G(x)=\frac{2 \mu}{\hbar^{2}} \int d k^{\prime} \frac{e^{i k^{\prime} x}}{k^{2}+k^{\prime 2}+i 0^{+}}=2 \pi \frac{i \mu}{\hbar^{2} k} e^{i k|x|}
$$

such that

$$
T_{S}\left(k, k^{\prime}\right)=\frac{1}{2 \pi} \frac{i k^{\prime} \frac{2 \hbar^{2}}{M}}{1-i k^{\prime} \frac{2 \hbar^{2}}{M g_{S}}} .
$$

In the presence of a quadratic Zeeman shift $Q$, there is a difference in modulus of incoming and outgoing wave 


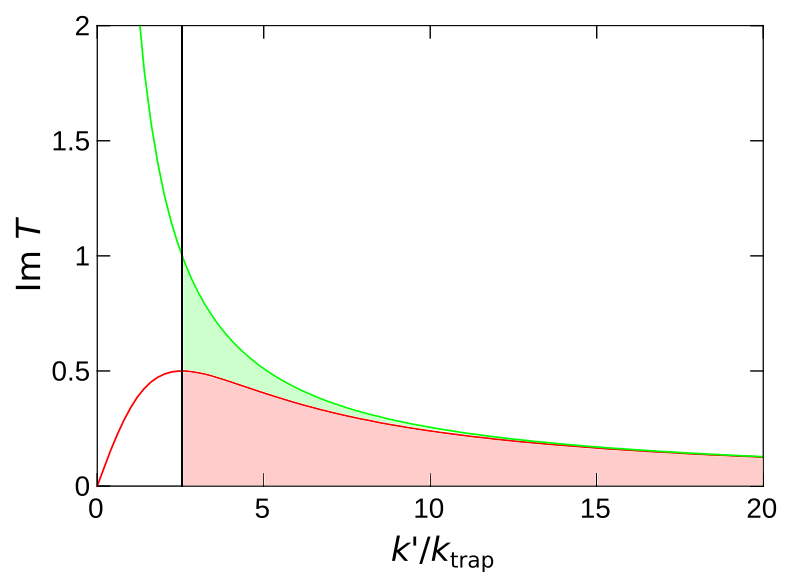

FIG. 8. Comparison of the imaginary part of the $T$-matrix [Eq. (D3)] (red line and region) with the expansion [Eq. (D4)] (green line and region) for a small coupling constant $g_{S}$. To avoid the singularity at $k^{\prime}=0$, we choose $T_{S}=0$ inside the region $\left|k^{\prime}\right| \leq M g_{S} / 2 \hbar^{2}$ indicated by the black line. The wave vector is scaled in terms of the trapping frequency $k_{\text {trap }}=\sqrt{M \omega / \hbar}$.

vectors $\left|k^{\prime}\right|=\sqrt{k^{2}+Q}$. Here and throughout this paper, if the argument of the square root becomes negative for a negative $Q$, the $T$-matrix vanishes and with it the entire collision term.

A problem absent in the $3 \mathrm{D}$ case is encountered during the low-energy expansion of Eq. (D2). The imaginary part of the $T$-matrix is given by

$$
\operatorname{Im} T_{S}\left(k, k^{\prime}\right)=\frac{1}{2 \pi} \frac{k^{\prime} \frac{2 \hbar^{2}}{M}}{1+\frac{k^{\prime 2} 4 \hbar^{4}}{M^{2} g_{S}^{2}}},
$$

and an expansion in powers of $g_{S}$ produces a singularity for $k^{\prime}=0$, since

$$
\operatorname{Im} T_{S}\left(k, k^{\prime}\right)=\frac{1}{2 \pi} \frac{g_{S}^{2} M}{2 \hbar^{2} k^{\prime}}+\cdots
$$

This singularity is artificial, and we use a cutoff to circumvent it. We choose the cutoff to be the maximum of $\operatorname{Im} T$ at $k^{\prime}=M g_{S} / 2 \hbar^{2}$, as depicted in Fig. 8. So, we use the expression

$$
T_{S}\left(k, k^{\prime}\right) \approx \frac{g_{S}}{2 \pi}- \begin{cases}0 & \text { if }\left|k^{\prime}\right|<\frac{M g_{S}}{2 \hbar^{2}} \\ \frac{i M g_{S}^{2}}{4 \pi \hbar^{2} k^{\prime}}+\cdots & \text { if }\left|k^{\prime}\right| \geq \frac{M g_{S}}{2 \hbar^{2}}\end{cases}
$$

to expand the $T$-matrix.

\section{APPENDIX E: SEMICLASSICAL GRADIENT EXPANSION}

In order to further simplify the expressions (C2), we assume the Wigner function to vary only slowly in space compared to single-particle wave functions. This assumption means that local contributions to the collision term dominate, and we perform a Taylor expansion for the spatial coordinate

$$
\begin{aligned}
& W_{i j}\left(x_{1}-\frac{r \pm r^{\prime}}{2}, p\right) \\
& \quad=W_{i j}\left(x_{1}, p\right)-\frac{r \pm r^{\prime}}{2} \partial_{x_{1}} W_{i j}\left(x_{1}, p\right)+\cdots ;
\end{aligned}
$$

therefore, the expansion of the product of Wigner functions in Eq. (C2) reads

$$
\begin{aligned}
W_{a c} & \left(x_{1}-\frac{r-r^{\prime}}{2}, p_{1}^{\prime}\right) W_{b d}\left(x_{1}-\frac{r+r^{\prime}}{2}, p_{2}^{\prime}\right) \\
= & W_{a c}\left(x_{1}, p_{1}^{\prime}\right) W_{b d}\left(x_{1}, p_{2}^{\prime}\right) \\
& -\frac{r}{2} \partial_{x_{1}}\left[W_{a c}\left(x_{1}, p_{1}^{\prime}\right) W_{b d}\left(x_{1}, p_{2}^{\prime}\right)\right] \\
& +\frac{r^{\prime}}{2} W_{b d}\left(x_{1}, p_{2}^{\prime}\right) \partial_{x_{1}} W_{a c}\left(x_{1}, p_{1}^{\prime}\right) \\
& -\frac{r^{\prime}}{2} W_{a c}\left(x_{1}, p_{1}^{\prime}\right) \partial_{x_{1}} W_{b d}\left(x_{1}, p_{2}^{\prime}\right)+\cdots
\end{aligned}
$$

Together with the expansion of the $T$-matrix above, we must be careful to expand the collision term in two small parameters in a meaningful way. One small parameter is the coupling constant proportional to the $s$-wave scattering length. The other one is related to the gradient expansion. Its magnitude is determined by the Fermi or thermal wavelength compared to the variation of the Wigner function determined by the system size. To maintain the unitarity of the $S$-matrix, we expand the $T$-matrix to second order. We thereby obtain terms linear in $a_{S}$ from $I_{i j}^{T}(x, p)$ and quadratic terms from $I_{i j}^{T}(x, p)$ and $I_{i j}^{T^{2}}(x, p)$. We expand the terms linear in $a_{S}$ up to first order in gradients and the terms quadratic in $a_{S}$ to zero order, keeping only the local term, which amounts to a semiclassical approximation of the theory. In this case, we substitute $W_{a c}\left(x_{1}-\left(\left(r-r^{\prime}\right) / 2\right), p_{1}^{\prime}\right)$ $W_{b d}\left(x_{1}-\left(\left(r+r^{\prime}\right) / 2\right), p_{2}^{\prime}\right) \approx W_{a c}\left(x_{1}, p_{1}^{\prime}\right) W_{b d}\left(x_{1}, p_{2}^{\prime}\right)$ into Eq. (C2), which means that further delta functions $\int d r^{\prime} e^{i\left(k_{2}-k_{1}\right) r^{\prime}}=2 \pi \delta\left(k_{2}-k_{1}\right) \quad$ and $\quad \int d r e^{i \kappa r}=2 \pi \delta(\kappa)$ appear. We introduce renamed variables $k_{ \pm} \rightarrow k, k_{1} \rightarrow k^{\prime}$, $x_{1}, p_{1} \rightarrow x, p$, and $p_{ \pm} \equiv p-\hbar\left(k \pm k^{\prime}\right)$; the local parts of the collision integral become

$$
\begin{aligned}
I_{i j}^{T}(x, p)= & \frac{-i 2 \pi}{\Delta t} \int d q \int d k^{\prime} \sum_{a b c d l} \delta\left(\epsilon_{k}-\epsilon_{k^{\prime}}+Q_{i l a b}\right) \\
& \times\left[\delta\left(k-k^{\prime}\right) \delta_{j c} \delta_{l d}-\delta\left(k+k^{\prime}\right) \delta_{l c} \delta_{j d}\right] \\
& \times\left(U_{i a l b}-\frac{i M \tilde{U}_{i a l b}}{\hbar^{2} k^{\prime}}\right) W_{a c}\left(x, p_{-}\right) W_{b d}\left(x, p_{+}\right) \\
& + \text {H.c. }
\end{aligned}
$$

and 


$$
\begin{aligned}
I_{i j}^{T^{2}}(x, p)= & \frac{2 \pi}{\Delta t} \int d q \int d k^{\prime} \sum_{a b c d l} \delta\left(\epsilon_{k}-\epsilon_{k^{\prime}}+Q_{i l a b}\right) \\
& \times \delta\left(\epsilon_{k^{\prime}}-\epsilon_{k}+Q_{c d j l}\right) U_{i a l b} U_{j c l d} W_{a c}\left(x, p_{-}\right) \\
& \times W_{b d}\left(x, p_{+}\right) .
\end{aligned}
$$

\section{APPENDIX F: SQUARES AND PRODUCTS OF DELTA FUNCTIONS}

In scattering theory, the square of a delta function of energy appears frequently, when terms quadratic in the $T$-matrix are involved. This term can be approximated as a well-known interpretation of this is $[\delta(E)]^{2} \approx$ $(\Delta t / 2 \pi \hbar) \delta(E)$, where $\Delta t$ denotes the elapsed time interval, which is quasi-infinite when compared to the duration of a single scattering event but nevertheless short compared to other relevant dynamics, like relaxation or the trapping period. This approximation is obtained by using the Fourier representation of the delta function

$$
\delta(E)=\frac{1}{2 \pi \hbar} \int d t e^{(i / \hbar) E t},
$$

such that

$$
\begin{aligned}
{[\delta(E)]^{2} } & =\delta(E) \frac{1}{2 \pi \hbar} \int d t e^{(i / \hbar) E t} \\
& =\delta(E) \frac{1}{2 \pi \hbar} \int d t \approx \delta(E) \frac{1}{2 \pi \hbar} \int_{\Delta t} d t=\frac{\Delta t}{2 \pi \hbar} \delta(E) .
\end{aligned}
$$

The same procedure can also be applied to products of the form $\delta\left(\epsilon_{k}-\epsilon_{k^{\prime}}\right) \delta\left(k-k^{\prime}\right)$ since

$$
\begin{aligned}
& \delta\left(\frac{\hbar^{2} k^{2}}{2 \mu}-\frac{\hbar^{2} k^{\prime 2}}{2 \mu}\right) \delta\left(k-k^{\prime}\right) \\
& =\frac{\mu}{\hbar^{2}\left|k^{\prime}\right|} \delta\left(|k|-\left|k^{\prime}\right|\right) \delta\left(|k|-\left|k^{\prime}\right|\right) \delta_{\operatorname{sgn}(k), \operatorname{sgn}\left(k^{\prime}\right)} \\
& =\frac{\hbar^{2}\left|k^{\prime}\right|}{\mu} \delta_{\operatorname{sgn}(k), \operatorname{sgn}\left(k^{\prime}\right)}\left[\delta\left(\frac{\hbar^{2} k^{2}}{2 \mu}-\frac{\hbar^{2} k^{\prime 2}}{2 \mu}\right)\right]^{2} \\
& \approx \frac{\hbar\left|k^{\prime}\right| \Delta t}{2 \pi \mu} \delta_{\operatorname{sgn}(k), \operatorname{sgn}\left(k^{\prime}\right)} \delta\left(\frac{\hbar^{2} k^{2}}{2 \mu}-\frac{\hbar^{2} k^{\prime 2}}{2 \mu}\right) \\
& =\frac{\Delta t}{2 \pi \hbar} \delta\left(k-k^{\prime}\right) .
\end{aligned}
$$

We modify this approximation to take into account the shift $Q$ in the quadratic Zeeman energy after a spin-changing collision. In our calculations, two situations appear. In the first, coming from Eq. (F3), there is only one shift and we must be careful that only the delta function with the shift comes from a $T$-matrix where we can approximate the integration area with the interval $\Delta t$ :

$$
\begin{aligned}
& \delta\left(\epsilon_{k}-\epsilon_{k^{\prime}}\right) \delta\left(\epsilon_{k}-\epsilon_{k^{\prime}}+Q\right) \\
& \quad \approx \delta\left(\epsilon_{k}-\epsilon_{k^{\prime}}\right) \frac{1}{2 \pi \hbar} \int_{\Delta t} d t e^{(i / \hbar)\left(\epsilon_{k}-\epsilon_{k^{\prime}}+Q\right) t} \\
& =\delta\left(\epsilon_{k}-\epsilon_{k^{\prime}}\right) \frac{1}{2 \pi \hbar} \int_{\Delta t} d t e^{(i / \hbar) Q t} \\
& =\frac{\Delta t}{2 \pi \hbar} \delta\left(\epsilon_{k}-\epsilon_{k^{\prime}}\right) \operatorname{sinc}\left(\frac{Q \Delta t}{2 \hbar}\right) .
\end{aligned}
$$

In the second case, both delta functions originate from the energy conservation of the $T$-matrix

$$
\begin{aligned}
\delta\left(\epsilon_{k}-\epsilon_{k^{\prime}}+Q_{1}\right) \delta\left(\epsilon_{k}-\epsilon_{k^{\prime}}+Q_{2}\right) & =\frac{1}{(2 \pi \hbar)^{2}} \int_{\Delta t} d t \int_{\Delta t} d t^{\prime} e^{(i / \hbar)\left(\epsilon_{k}-\epsilon_{k^{\prime}}+Q_{1}\right) t} e^{(i / \hbar)\left(\epsilon_{k}-\epsilon_{k^{\prime}}+Q_{2}\right) t^{\prime}} \\
& =\frac{1}{(2 \pi \hbar)^{2}} \int_{\Delta t} d t \int_{\Delta t} d t^{\prime} e^{(i / \hbar)\left[\left(\epsilon_{k}-\epsilon_{k^{\prime}}\right)\left(t+t^{\prime}\right)+Q_{1} t+Q_{2} t^{\prime}\right]} \\
& =\frac{2}{(2 \pi \hbar)^{2}} \int_{\Delta t} d u \int_{\Delta t} d u^{\prime} e^{(i / \hbar)\left(\epsilon_{k}-\epsilon_{k^{\prime}}\right) u} e^{\left(i Q_{1} / 2 \hbar\right)\left(u-u^{\prime}\right)} e^{\left(i Q_{2} / 2 \hbar\right)\left(u+u^{\prime}\right)} \\
& =\frac{2}{(2 \pi \hbar)^{2}} \int_{\Delta t} d u \int_{\Delta t} d u^{\prime} e^{(i / \hbar)\left[\epsilon_{k}-\epsilon_{k^{\prime}}+\left(Q_{1}+Q_{2} / 2\right)\right] u} e^{(i / 2 \hbar)\left(Q_{2}-Q_{1}\right) u^{\prime}} \\
& \approx \frac{\Delta t}{2 \pi \hbar} \delta\left(\epsilon_{k}-\epsilon_{k^{\prime}}+\frac{1}{2}\left(Q_{1}+Q_{2}\right)\right) \operatorname{sinc}\left(\frac{Q_{2}-Q_{1}}{2 \hbar} \Delta t\right) .
\end{aligned}
$$

The time interval $\Delta t$ that appears in front cancels with the one introduced at the beginning [Eq. (18)], and for the sinc function, we assume it to be small such that sinc $\rightarrow 1$.

\section{APPENDIX G: SINGLE-MODE APPROXIMATION WITH THE QZE AND IN 1D}

Taking the quadratic Zeeman effect into account, the expressions for the single-mode approximation [Eq. (9)] become slightly more complicated. The equation of motion is now given by 


$$
\frac{d}{d t} M_{m n}=-\frac{M}{4 \pi \hbar^{4}}\left\{\sum_{a b l}\left(\lambda_{\text {mlab }}^{(1)} \tilde{U}_{\text {malb }}^{\prime} M_{a n} M_{b l}+\lambda_{\text {nlab }}^{(1)} \tilde{U}_{\text {nalb }}^{\prime} M_{m a} M_{l b}\right)-\sum_{a b c d l} \lambda_{\text {mnlabcd }}^{\prime(2)} U_{m a l b}^{\prime} U_{n c l d}^{\prime} M_{a c} M_{b d}\right\},
$$

where the two now separate integrals $\lambda^{(1,2)}$ are spin dependent and given by

$$
\lambda_{a b c d}^{\prime(1)}=\frac{1}{N} \int d \boldsymbol{r} \int d \boldsymbol{p} \int d \boldsymbol{q} \sqrt{\boldsymbol{q}^{2}+\Delta_{a b c d}} f_{0}(\boldsymbol{r}, \boldsymbol{p}) f_{0}(\boldsymbol{r}, \boldsymbol{p}-\boldsymbol{q})
$$

and

$$
\lambda_{m n l a b c d}^{\prime(2)}=\frac{1}{N} \int d \boldsymbol{r} \int d \boldsymbol{p} \int d \boldsymbol{q} \sqrt{\boldsymbol{q}^{2}+\Delta_{\text {mnlabcd }}} f_{0}(\boldsymbol{r}, \boldsymbol{p}) f_{0}(\boldsymbol{r}, \boldsymbol{p}-\boldsymbol{q}) .
$$

The single-mode approximation can also be applied to the 1D system, from the Boltzmann equation (4). Equation (G1) changes to

$$
\frac{d}{d t} M_{m n}=-\frac{M}{\hbar^{2}}\left\{\sum_{a b l}\left(\lambda_{m l a b}^{(1)} \tilde{U}_{m a l b} M_{a n} M_{b l}+\lambda_{n l a b}^{(1)} \tilde{U}_{n a l b} M_{m a} M_{l b}\right)-\sum_{a b c d l} \lambda_{m n l a b c d}^{(2)} U_{m a l b} U_{n c l d} M_{a c} M_{b d}\right\},
$$

and the other expressions become

$$
\lambda_{a b c d}^{(1)}=\frac{1}{N} \int d x \int d p \int_{q^{2}>\epsilon_{1,2}} d q \frac{f_{0}(r, p) f_{0}(r, p-q)}{\sqrt{q^{2}+\Delta_{a b c d}}}
$$

and

$$
\lambda_{\text {mnlabcd }}^{(2)}=\frac{1}{N} \int d x \int d p \int_{q^{2}>\epsilon_{3}} d q \frac{f_{0}(r, p) f_{0}(r, p-q)}{\sqrt{q^{2}+\Delta_{\text {mnlabcd }}}},
$$

respectively, where the infrared cutoff described in Appendix D must be employed. The equilibrium distribution is given by

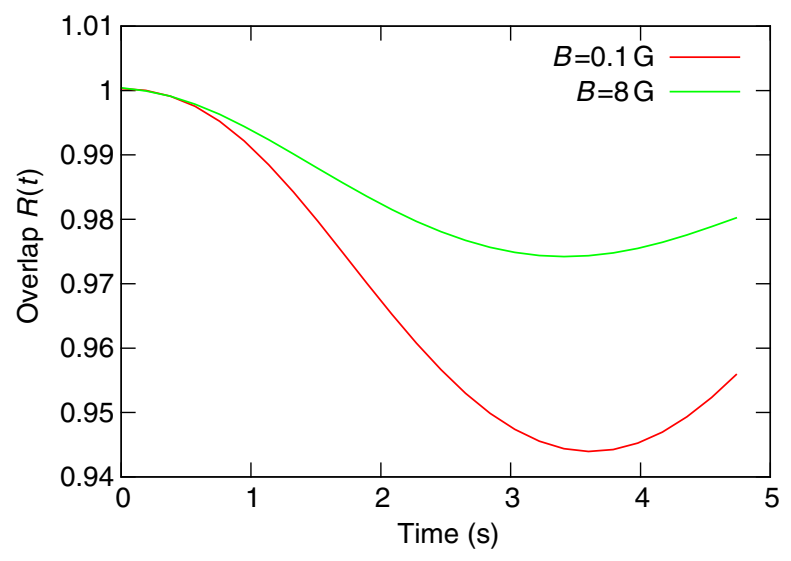

FIG. 9. Overlap between a noninteracting equilibrium distribution [Eq. (G7)] and the Wigner function during the simulations performed to obtain the temperatures in Fig. 7(b).

$$
f_{0}(x, p)=\left\{\exp \left[\frac{1}{k_{B} T}\left(\frac{p^{2}}{2 M}+\frac{1}{2} M \omega^{2} x^{2}-\mu\right)\right]+1\right\}^{-1} .
$$

A comparison of 1D single-mode results with the full 1D Boltzmann equation shows good agreement for pure spin relaxation, as shown in Fig. 4.

A further inclusion of the coherent oscillations described by the commutator in Eq. (4) into the single-mode equation shows that while the oscillations themselves are reproduced with high accuracy [36], the damping of coherent oscillations such as in Fig. 3 is not captured well. We attribute this feature to the fact that damping is driven by the much stronger spin-conserving collisions and affects more strongly the individual phase-space distributions of the spin states, which are taken to be constant in time in the single-mode approximation. Thus, we consider it necessary to use the full 1D Boltzmann in these cases.

\section{APPENDIX H: CONCEPT OF TEMPERATURE IN FIG. 7}

Under the assumptions stated at the end of Sec. III, we extract a temperature from our 1D numerical simulations as follows. At each time, we have the full Wigner function available and can compare the Wigner function of, e.g., the $m=1 / 2$ component $W_{(1 / 2)(1 / 2)}(x, p, t) \equiv W(x, p, t)$ to a noninteracting equilibrium distribution $f_{0}(x, p)$ [Eq. (G7)]. This distribution is determined by particle number

$$
N=\int d x \int d p f_{0}(x, p)
$$

and trap energy 


$$
E=\int d x \int d p\left(\frac{p^{2}}{2 M}+\frac{1}{2} M \omega^{2} x^{2}\right) f_{0}(x, p)
$$

but also equivalently by temperature and chemical potential. Hence, we calculate at each time the particle number and trap energy of $W(x, p, t)$ and generate a Fermi distribution $\quad f_{0}(x, p, t)=f_{0}[N(t), E(t)]=f_{0}[\mu(t), T(t)]$ with the same values for $N$ and $E$. The temperature of this distribution is plotted in Fig. 7(b) as an estimate for the temperature of $W$. The overlap between this equilibrium distribution and the Wigner function

$$
R(t)=\frac{\int d x \int d p f_{0}(x, p, t) W_{(1 / 2)(1 / 2)}(x, p, t)}{\int d x \int d p W_{(1 / 2)(1 / 2)}(x, p, t) W_{(1 / 2)(1 / 2)}(x, p, t)}
$$

is plotted in Fig. 9 and for the times we consider maintains sufficiently large values.

[1] M. Srednicki, Chaos and Quantum Thermalization, Phys. Rev. E 50, 888 (1994).

[2] M. Rigol, V. Dunjko, and M. Olshanii, Thermalization and Its Mechanism for Generic Isolated Quantum Systems, Nature (London) 452, 854 (2008).

[3] J. Dziarmaga, Dynamics of a Quantum Phase Transition and Relaxation to a Steady State, Adv. Phys. 59, 1063 (2010).

[4] A. Polkovnikov, K. Sengupta, A. Silva, and M. Vengalattore, Nonequilibrium Dynamics of Closed Interacting Quantum Systems, Rev. Mod. Phys. 83, 863 (2011).

[5] M. Gring, M. Kuhnert, T. Langen, T. Kitagawa, B. Rauer, M. Schreitl, I. Mazets, D. A. Smith, E. Demler, and J. Schmiedmayer, Relaxation and Prethermalization in an Isolated Quantum System, Science 337, 1318 (2012).

[6] T. Langen, R. Geiger, M. Kuhnert, B. Rauer, and J. Schmiedmayer, Local Emergence of Thermal Correlations in an Isolated Quantum Many-Body System, Nat. Phys. 9, 640 (2013).

[7] J. Berges, S. Borsányi, and C. Wetterich, Prethermalization, Phys. Rev. Lett. 93, 142002 (2004).

[8] T. Kinoshita, T. Wenger, and D. S. Weiss, A Quantum Newton's Cradle, Nature (London) 440, 900 (2006).

[9] S. Hofferberth, I. Lesanovsky, B. Fischer, T. Schumm, and J. Schmiedmayer, Non-equilibrium Coherence Dynamics in One-Dimensional Bose Gases, Nature (London) 449, 324 (2007).

[10] M. Cheneau, P. Barmettler, D. Poletti, M. Endres, P. Schauß, T. Fukuhara, C. Gross, I. Bloch, C. Kollath, and S. Kuhr, Light-Cone-like Spreading of Correlations in a Quantum Many-Body System, Nature (London) 481, 484 (2012).

[11] S. Trotzky, Y.-A. Chen, A. Flesch, I. P. McCulloch, U. Schollwöck, J. Eisert, and I. Bloch, Probing the Relaxation towards Equilibrium in an Isolated Strongly Correlated One-Dimensional Bose Gas, Nat. Phys. 8, 325 (2012).
[12] J. Lux, J. Müller, A. Mitra, and A. Rosch, Hydrodynamic Long-Time Tails after a Quantum Quench, arXiv:1311.7644.

[13] M. Erhard, H. Schmaljohann, J. Kronjäger, K. Bongs, and K. Sengstock, Bose-Einstein Condensation at Constant Temperature, Phys. Rev. A 70, 031602 (2004).

[14] L. E. Sadler, J. M. Higbie, S. R. Leslie, M. Vengalattore, and D. M. Stamper-Kurn, Spontaneous Symmetry Breaking in a Quenched Ferromagnetic Spinor BoseEinstein Condensate, Nature (London) 443, 312 (2006).

[15] A. Griesmaier, J. Werner, S. Hensler, J. Stuhler, and T. Pfau, Bose-Einstein Condensation of Chromium, Phys. Rev. Lett. 94, 160401 (2005).

[16] H. Schmaljohann, M. Erhard, J. Kronjäger, M. Kottge, S. van Staa, L. Cacciapuoti, J. J. Arlt, K. Bongs, and K. Sengstock, Dynamics of $F=2$ Spinor Bose-Einstein Condensates, Phys. Rev. Lett. 92, 040402 (2004).

[17] C. Klempt, O. Topic, G. Gebreyesus, M. Scherer, T. Henninger, P. Hyllus, W. Ertmer, L. Santos, and J. J. Arlt, Multiresonant Spinor Dynamics in a BoseEinstein Condensate, Phys. Rev. Lett. 103, 195302 (2009).

[18] J. Kronjäger, C. Becker, P. Soltan-Panahi, K. Bongs, and K. Sengstock, Spontaneous Pattern Formation in an Antiferromagnetic Quantum Gas, Phys. Rev. Lett. 105, 090402 (2010).

[19] J. Mur-Petit, M. Guilleumas, A. Polls, A. Sanpera, M. Lewenstein, K. Bongs, and K. Sengstock, Dynamics of $F=1{ }^{87} \mathrm{Rb}$ Condensates at Finite Temperatures, Phys. Rev. A 73, 013629 (2006).

[20] L. Santos and T. Pfau, Spin-3 Chromium Bose-Einstein Condensates, Phys. Rev. Lett. 96, 190404 (2006).

[21] D. M. Stamper-Kurn and M. Ueda, Spinor Bose Gases: Explorations of Symmetries, Magnetism and Quantum Dynamics, Rev. Mod. Phys. 85, 1191 (2013).

[22] H. K. Pechkis, J. P. Wrubel, A. Schwettmann, P. F. Griffin, R. Barnett, E. Tiesinga, and P. D. Lett, Spinor Dynamics in an Antiferromagnetic Spin-1 Thermal Bose Gas, Phys. Rev. Lett. 111, 025301 (2013).

[23] C. A. Regal, M. Greiner, and D. S. Jin, Observation of Resonance Condensation of Fermionic Atom Pairs, Phys. Rev. Lett. 92, 040403 (2004).

[24] M. W. Zwierlein, J. R. Abo-Shaeer, A. Schirotzek, C. H. Schunck, and W. Ketterle, Vortices and Superfluidity in a Strongly Interacting Fermi Gas, Nature (London) 435 , 1047 (2005).

[25] A. Sommer, M. Ku, G. Roati, and M. W. Zwierlein, Universal Spin Transport in a Strongly Interacting Fermi Gas, Nature (London) 472, 201 (2011).

[26] M. Koschorreck, D. Pertot, E. Vogt, and M. Köhl, Universal Spin Dynamics in Two-Dimensional Fermi Gases, Nat. Phys. 9, 405 (2013).

[27] X. Du, L. Luo, B. Clancy, and J. E. Thomas, Observation of Anomalous Spin Segregation in a Trapped Fermi Gas, Phys. Rev. Lett. 101, 150401 (2008).

[28] S. S. Natu, and E. J. Mueller, Anomalous Spin Segregation in a Weakly Interacting Two-Component Fermi Gas, Phys. Rev. A 79, 051601(R) (2009).

[29] G. B. Jo, Y. R. Lee, J. H. Choil, C. A. Christensen, T. H. Kim, J. H. Thywissen, D. E. Pritchard, and W. Ketterle, Itinerant Ferromagnetism in a Fermi Gas of Ultracold Atoms, Science 325, 1521 (2009). 
[30] G. J. Conduit and E. Altman, Effect of Three-Body Loss on Itinerant Ferromagnetism in an Atomic Fermi Gas, Phys. Rev. A 83, 043618 (2011).

[31] S. Zhang and T.-L. Ho, Atom Loss Maximum in Ultra-cold Fermi Gases, New J. Phys. 13, 055003 (2011).

[32] D. Pekker, M. Babadi, R. Sensarma, N. Zinner, L. Pollet, M. W. Zwierlein, and E. Demler, Competition between Pairing and Ferromagnetic Instabilities in Ultracold Fermi Gases near Feshbach Resonances, Phys. Rev. Lett. 106, 050402 (2011).

[33] J. S. Krauser, J. Heinze, N. Fläschner, S. Götze, O. Jürgensen, D.-S. Lühmann, C. Becker, and K. Sengstock, Coherent Multi-flavour Spin Dynamics in a Fermionic Quantum Gas, Nat. Phys. 8, 813 (2012).

[34] Y. Dong and H. Pu, Spin Mixing in Spinor Fermi Gases, Phys. Rev. A 87, 043610 (2013).

[35] J. Heinze, J. S. Krauser, N. Fläschner, K. Sengstock, C. Becker, U. Ebling, A. Eckardt, and M. Lewenstein, Engineering Spin-Waves in a High-Spin Ultracold Fermi Gas, Phys. Rev. Lett. 110, 250402 (2013).

[36] J. S. Krauser, U. Ebling, N. Fläschner, J. Heinze, K. Sengstock, M. Lewenstein, A. Eckardt, and C. Becker, Giant Spin Oscillations in an Ultracold Fermi Sea, Science 343, 157 (2014).

[37] M. R. Gallis and G. N. Fleming, Environmental and Spontaneous Localization, Phys. Rev. A 42, 38 (1990).

[38] K. Hornberger and J.E. Sipe, Collisional Decoherence Reexamined, Phys. Rev. A 68, 012105 (2003).

[39] L. R. Corruccini, D. D. Osheroff, D. M. Lee, and R. C. Richardson, Spin-Wave Phenomena in Liquid ${ }^{3} \mathrm{He}$ Systems, J. Low Temp. Phys. 8, 229 (1972).

[40] B. R. Johnson, J. S. Denker, N. Bigelow, L. P. Lévy, J. H. Freed, and D. M. Lee, Observation of Nuclear Spin Waves in Spin-Polarized Atomic Hydrogen Gas, Phys. Rev. Lett. 52, 1508 (1984).

[41] E. P. Bashkin, Spin Waves in Polarized Paramagnetic Gases, JETP Lett. 33, 8 (1981).

[42] L. P. Lévy and A. E. Ruckenstein, Collective Spin Oscillations in Spin-Polarized Gases: Spin-Polarized Hydrogen, Phys. Rev. Lett. 52, 1512 (1984).
[43] C. Lhuillier and F. Laloë, Transport Properties in a Spin Polarized Gas, I, J. Phys. (Paris) 43, 197 (1982).

[44] C. Lhuillier and F. Laloë, Transport Properties in a Spin Polarized Gas, II, J. Phys. (Paris) 43, 225 (1982).

[45] J. R. Owers-Bradley, Spin-Polarized ${ }^{3} \mathrm{He}-{ }^{4} \mathrm{He}$ Liquids, Rep. Prog. Phys. 60, 1173 (1997).

[46] J. N. Fuchs, D. M. Gangardt, and F. Laloë, Large Amplitude Spin Waves in Ultra-cold Gases, Eur. Phys. J. D 25, 57 (2003).

[47] F. Piéchon, J. N. Fuchs, and F. Laloë, Cumulative Identical Spin Rotation Effects in Collisionless Trapped Atomic Gases, Phys. Rev. Lett. 102, 215301 (2009).

[48] U. Ebling, A. Eckardt, and M. Lewenstein, Spin Segregation via Dynamically Induced Long-Range Interactions in a System of Ultracold Fermions, Phys. Rev. A 84, 063607 (2011).

[49] C. D. Fertig, K. M. O’Hara, J. H. Huckans, S. L. Rolston, W. D. Phillips, and J. V. Porto, Strongly Inhibited Transport of a Degenerate 1D Bose Gas in a Lattice, Phys. Rev. Lett. 94, 120403 (2005).

[50] E. Timmermans, Degenerate Fermion Gas Heating by Hole Creation, Phys. Rev. Lett. 87, 240403 (2001).

[51] S. S. Natu and E. J. Mueller, Spin Waves in a Spin-1 Bose Gas, Phys. Rev. A 81, 053617 (2010).

[52] G. M. Kavoulakis, C. J. Pethick, and H. Smith, Relaxation Processes in Clouds of Trapped Bosons above the BoseEinstein Condensation Temperature, Phys. Rev. Lett. 81, 4036 (1998).

[53] P. Massignan, G. M. Bruun, and H. Smith, Viscous Relaxation and Collective Oscillations in a Trapped Fermi Gas near the Unitarity Limit, Phys. Rev. A 71, 033607 (2005).

[54] G. Breit and I. I. Rabi, Measurement of Nuclear Spin, Phys. Rev. 38, 2082 (1931).

[55] E. Arimondo, M. Inguscio, and P. Violino, Experimental Determinations of the Hyperfine Structure in the Alkali Atoms, Rev. Mod. Phys. 49, 31 (1977).

[56] M. Olshanii, Atomic Scattering in the Presence of an External Confinement and a Gas of Impenetrable Bosons, Phys. Rev. Lett. 81, 938 (1998). 